Pacific Journal of Mathematics

THE FACTORS OF THE RAMIFICATION SEQUENCE OF A
CLASS OF WILLY RAMIFIED $v$-RINGS 


\title{
THE FACTORS OF THE RAMIFICATION SEQUENCE OF A CLASS OF WILDLY RAMIFIED $v$-RINGS
}

\author{
ROBERT D. DAVIS
}

\begin{abstract}
Let $R_{e}$ denote a $v$-ring of characteristic 0 , with maximal ideal $M$ and residue field $h$ of characteristic $p(p \neq 0,2)$ in which $p$ generates the eth power of the maximal ideal. If $p$ divides $e, R_{e}$ is said to be wildly ramified. This work is concerned primarily with the determination of the factor groups of the ramification sequences of wildly ramified $v$-rings having ramification $2 p$.
\end{abstract}

The canonical homomorphisms of the ramification sequence are used to show that in all except $G_{1} / H_{1}$ the successive factor groups are isomorphic to subgroups of the additive group of the residue field or to subgroups of the additive group of derivations on the residue field. Then the Eisenstein polynomial of $R_{2 p}$ over $R$ is used to determine bounds on the range of the the canonical homomorphism. One then constructs inertial automorphisms, using convergent higher derivations to establish that those bounds do, in fact, describe the range. Further, it is found that if $G_{1} / H_{1}$ is nontrivial, it is isomorphic to the group of order 2 , and that $G_{1} / H_{1}$ contains the first known examples of $v$ rings having inertial automorphisms which are neither derivation automorphisms nor automorphisms of finite order. In addition the Galois theory of totally ramified extensions $R_{p q}(q<p)$ is treated. Necessary and sufficient conditions for $R_{p q} / R$ to be Galois are found as well as the location of the Galois maps in the ramification sequence.

The determination of the factors of the ramification sequence extends the work of MacLane [8], Heerema [4] and Neggers [9]. The Galois theory of totally ramified extensions $R_{p q}(q<p)$ of an unramified $v$-ring, treated in §III generalizes the work of Wishart [13] and Davis and Wishart [1]. The convergent higher derivation used here as in the work of Heerema is completely described in [5], so a discussion of it will not be included.

In addition to evaluating the factor groups of the ramification sequence, a second object of this work was to determine the relationship of the subgroup of derivation automorphisms $G_{D}$ to the ramification sequence, where $\alpha \in G_{D}$ if there exists a convergent higher derivation $D=\left\{D_{j}\right\}$ such that $\alpha=\sum_{j=0}^{\infty} D_{j}$. In earlier work Neggers [9, Theorems 4 and 5] has shown that for arbitrary $e$, if $i \geqq$ $(e+p) /(p-1), G_{i} \subset G_{D}$ and that for $i, j \geqq(e+p) /(p-1), G_{i} / G_{i+1} \cong$ $G_{j} / G_{j+1}$ and $H_{i} / G_{i+1} \cong H_{j} / G_{j+1}$. He also characterized these factor groups in terms of derivations [9, Theorem 6]. Until now in every 
known case of complete local rings that have been investigated, it has been found that the group of inertial automorphisms is generated by the automorphisms of finite order and $G_{D}$. However, in the proof of Theorem 3 we exhibit automorphisms that can be neither derivation automorphisms, nor automorphisms of finite order, nor composites of the two.

Let $G$ be the group of automorphisms of $R_{e}$, and let $M$ be the maximal ideal whose $j$ th power will be denoted throughout by $M(j)$; the residue field is $h \cong R_{e} / M$. The subgroup $G_{1}$ of automorphisms which induce the identity map on $h$ is called the inertial automorphism group of $R_{e}$. A chain of normal subgroups of $G_{1}$ is given by the following:

$$
\begin{aligned}
G_{i} & =\left\{\alpha \in G_{1} \mid \alpha(a)-a \in M(i) \text { for every } a \in R_{e}\right\} \\
H_{i} & =\left\{\alpha \in G_{i} \mid \alpha(a)-a \in M(i+1) \text { for every } a \in M\right\}
\end{aligned}
$$

so that

$$
G_{1} \supseteqq H_{1} \supseteqq G_{2} \supseteqq H_{2} \cdots \text {. }
$$

This chain of subgroups is known as the ramification sequence of $R_{e}$.

To stabilize the notation, we will hereinafter denote by $V(a)$ the exponential valuation of an element $a \in R_{e}$; we denote by either $\rho(a)$ or $\bar{a}$ the image of $a \in R_{e}$ in the residue field $h$ under the natural map of $R$ onto $h$; we will assume that $h$ is not perfect, i.e., that $h$ has a nontrivial $p$-basis, since otherwise $H_{i}=G_{i+1}$; and we will always assume that the prime $p \neq 2$. In addition the minimum polynomial of $R_{2 p} / R$ will always be

$$
f(x)=x^{2 p}+p \sum_{i=0}^{2 p-1} a_{i} x^{i}
$$

and $s$ will always denote the least positive integer for which $a_{s}$ is a unit in (1.1). In case no $a_{i}$ is a unit, we will say that $s=2 p$.

Letting $\pi$ denote a prime element for $R_{2 p}$, observe that $\pi$ always satisfies an equation of the form

$$
\pi^{2 p}+p u=0,
$$

where $u$ is a unit in $R_{2 p}$ such that $\bar{u}=\bar{a}_{0}$. Moreover, if $\bar{u} \in h^{p}$, then a $v \in R_{2 p}$ may be chosen so that $\pi$ satisfies an equation of the form

$$
\pi^{2 p}+p\left(v^{p}+\pi^{s} w\right)=0
$$

where $\bar{v}^{p}=\bar{a}_{0}$. Note that the value of $s$ as well as whether $\bar{a}_{0} \in h^{p}$ is independent of the choice of $\pi$. Further, it will be shown in Lemma 1.2 that $\pi$ can be chosen so that $\bar{v} \in h^{p}$. If $s=2 p-1$ and $a_{0} \in h^{p}$, the form of (1.3) can be modified to 


$$
\pi^{2 p}+p\left(v^{p}+\pi^{2 p-1} w_{0}+p w_{1}\right)=0
$$

in which $\bar{w}_{0}=\bar{a}_{2 p-1}$.

Using $u, v, w$, and $w_{1}$ as in (1.2), (1.3), and (1.4) and assuming $\bar{v} \in h^{p}$ we define the following sets of derivations on $h$.

$\mathscr{D}(h)=$ Group of all derivations on $h$.

$\mathscr{D}_{0}(h)=$ Group of $\delta \in \mathscr{D}(h)$ such that $\delta(\bar{u})=0$.

$\mathscr{D}_{1}(h)=$ Group of $\delta \in \mathscr{D}(h)$ such that $\delta(\bar{w})=0$.

$\mathscr{D}_{2}(h)=$ Group of $\delta \in \mathscr{D}(h)$ such that $\delta(\bar{w})=\rho\left(2 v^{p} b^{p}+w b\right)$ for some $\bar{b} \in h$.

$\mathscr{D}_{3}(h)=$ Group of $\delta \in \mathscr{D}(h)$ such that $\delta(\bar{w})=\rho\left(2 v^{p} b^{p}+2 w b\right)$ for some $\bar{b} \in h$.

$\mathscr{D}_{4}(h)=$ Group of $\delta \in \mathscr{D}(h)$ such that $\delta(\bar{w}) \in h^{p}$.

$\mathscr{D}_{5}(h)=$ Group of $\delta \in \mathscr{D}(h)$ such that $\delta\left(\bar{w}_{1}\right)=0$.

$\mathscr{D}_{\theta}(h)=$ Group of $\delta \in \mathscr{D}(h)$ such that $\delta(\bar{w})=\rho\left(2 v^{p} b-2 v^{2 p} b^{p}\right)$ for some $\bar{b} \in h$.

We can now describe the factor groups $H_{i} / G_{i+1}$ and $G_{i} / H_{i}$ in every case in the following theorems:

THEOREM 1. If $\bar{u} \notin h^{p}$, then $H_{i} / G_{i+1} \cong \mathscr{D}_{0}(h)$. If $\bar{u} \in h^{p}$, then $H_{i} / G_{i+1}$ is given in the table below.

TABLE I

\begin{tabular}{l|c|c|c|c|c|c|c|c}
\hline & \multicolumn{2}{|c|}{$\phi_{1}\left(H_{1}\right) \cong H_{1} / G^{2}$} & \multicolumn{2}{|c|}{$\phi_{2}\left(H_{2}\right) \cong H_{2} / G_{3}$} & $\phi_{3}\left(H_{3}\right) \cong H_{3} / G_{4}$ & $\cdots$ \\
\hline & $\bar{w} \in h^{p}$ & $\bar{w} \notin h^{p}$ & $\bar{w} € h^{p}$ & $\bar{w} \notin h^{p}$ & & \\
\hline $0<s<p-1$ & $\mathscr{D}(h)$ & $\mathscr{D}(h)$ & $\mathscr{D}(h)$ & $\mathscr{D}(h)$ & $\mathscr{D}(h)$ & & $\cdots$ \\
$s=p-1$ & $\mathscr{D}(h)$ & $\mathscr{D}_{2}(h)$ & $\mathscr{D}(h)$ & $\mathscr{D}(h)$ & $\mathscr{D}(h)$ & & $\ldots$ \\
$s=p$ & $*$ & $\mathscr{D}_{1}(h)$ & $*$ & $\mathscr{D}_{1}(h)$ & $*$ & & $\ldots$ \\
$p<s<2 p-2$ & $\mathscr{D}(h)$ & $\mathscr{D}_{1}(h)$ & $\mathscr{D}(h)$ & $\mathscr{D}) h)$ & $\mathscr{D}(h)$ & & $\ldots$ \\
$s=2 p-2$ & $\mathscr{D}(h)$ & $\mathscr{D}_{1}(h)$ & $\mathscr{D}(h)$ & $\mathscr{D}_{3}(h)$ & $\mathscr{D}(h)$ & & $\ldots$ \\
$s=2 p-1$ & $\mathscr{D}_{5}(h)$ & $\mathscr{D}_{4}(h)$ & $\mathscr{D}(h)$ & $\mathscr{D}_{1}(h)$ & $\mathscr{D}(h)$ & & $\ldots$ \\
$s=2 p$ & $\mathscr{D}(h)$ & $\mathscr{D}_{1}(h)$ & $\mathscr{D}(h)$ & $\mathscr{D}_{1}(h)$ & $\mathscr{D}(h)$ & + & $\ldots$ \\
\hline
\end{tabular}

* Let $\sigma$ be the smallest integer greater than $p$ for which $a_{\sigma}$ is a unit, or if $a_{i} \epsilon$ $M(2 p)$ for $i=p+1, \cdots, 2 p-1$, then $\sigma=2 p$. If $\bar{a}_{p} \in h^{p}$, then $H_{i} / G_{+1}$ is given by the row in which $s=\sigma$ and the column for a given $i$ obtained by letting $\bar{a}_{\sigma}$ assume the role of $\bar{w}$.

$\dagger$ If $\bar{w} \notin h^{p}$ and $p=3$, then $H_{3} / G_{4} \cong \mathscr{D}_{8}(h)$.

THEOREM 2. Let $h^{+}$denote the additive group of $h$. Let $i>1$ and for $\alpha \in G_{i}$, define $\psi_{i}(\alpha)=\rho\left([\alpha(\pi)-\pi] / \pi^{i}\right)$. If $\bar{u} \notin h^{p}$, then $\psi_{i}\left(G_{i}\right) \cong$ $G_{i} / H_{i} \cong h^{+}$. If for $\bar{u} \in h^{p}$, we have $s \neq p$, or $s=p$ and $\bar{w} \in h^{p}$, then $G_{i} \neq H_{i}$ if and only if $R_{2 p} / R$ is Galois and $i$ is equal to the $n$ of the theorem in [1]. In this case $G_{i} / H_{i}$ is the group of order $p$. When $s=p$ and $\bar{w} \notin h^{p}, G_{2}=H_{2}$ and $\psi_{i}\left(G_{i}\right) \cong G_{i} / H_{i} \cong h^{+}$for $i>2$. If $G_{1} \neq H_{1}$, then $G_{1} / H_{1}$ is isomorphic to the group of order 2 . 
TheOREM 3. Suppose that in (1.1) we let $t=\operatorname{Min}\left\{V\left(a_{i}\right) / 2 p \mid i=\right.$ $1,2, \cdots, 2 p-1\}, j=\operatorname{Min}\left\{i \mid V\left(a_{i}\right)=2 t p\right\}$. Further, if $\bar{a}_{0} \in h^{p}$, let $a_{0}=$ $c_{0}^{p}+c_{1} p$ for $c_{0}, c_{1} \in R$. Then $G_{1} \neq H_{1}$ except when $\bar{a}_{0} \in h^{p}$ in the following cases:

(a) $t=0$ and $j$ is odd unless $j=p$ and $\bar{a}_{p} \in h^{p}$.

(b) $t=0, j=p-1, \bar{a}_{p-1} \in h^{p}, \bar{a}_{p} \neq 0$ and $R_{2 p} / R$ is not Galois.

(c) $t=1, j=1$ and $\bar{c}_{1} \in h^{p}$.

To prove these we need to state a few basic results, some of which are proved elsewhere.

In what follows, if $T$ is a subring of $R_{e}$, the symbols $\mathscr{C}\left(T, R_{e}\right)$, $\mathscr{C}_{c}\left(T, R_{e}\right)$, and $\mathscr{C}_{u}\left(T, R_{e}\right)$ will denote the set of higher derivations, convergent higher derivations, and uniformly convergent higher derivations respectively, having domain $T$ and range $R_{e}$. See [5, Definition 3] for definitions of these.

For convenience we state here the following two results of Heerema.

Theorem A [5, Theorem 4]. Let $\bar{S}$ be a p-basis for $h$ and let $S \subset R$ be a set of representatives of the elements of $\bar{S}$. If $I$ is the set of positive integers and $f$ is a mapping from $S \times I$ into $R_{e}$, then there is one and only one $D \in \mathscr{L}\left(R, R_{e}\right)$ such that $D_{i}(\xi)=f(\xi, i)$ for all $\xi \in S$ and $i \in I$. Moreoever, $D$ converges (uniformly) if and only if $D$ converges (uniformly) on $S$.

LEMma A [4, Lemma 1]. If $S$ is a set of representatives in $R$ of a p-basis $\bar{S}$ for $h$ and $D \in \mathscr{H}\left(R, R_{e}\right)$ is such that $D_{j}(S) \subset M\left(t_{j}\right) \subset$ $M, j \geqq 1$, then $D_{i}(R) \subset M\left(q_{i}\right)$ where

$$
q_{i}=\min \left\{t_{j_{1}}+\cdots+t_{j_{i}} \mid i \geqq 1, j_{1}+\cdots+j_{i}=i \text {, and } t_{0}=0\right\} \text {. }
$$

Now suppose that $D=\left\{D_{i}\right\} \in \mathscr{C}\left(R, R_{e}\right)$. Then $D$ extends uniquely to $D \in \mathscr{C}\left(R_{e}, K_{e}\right)$ where $K_{e}$ is the quotient field of $R_{e}$. Moreover, $D \in \mathscr{H}\left(R_{e}, R_{e}\right)$ if and only if $D(\pi) \in R_{e}$, and if $D \in \mathscr{H}_{c}\left(R, R_{e}\right)$, then $D$ extends to $D \in \mathscr{K}_{c}\left(R_{e}, R_{e}\right)$ if and only if $D(\pi)$ converges. The extension of each $D_{i}$ in $D \in \mathscr{H}\left(R, R_{e}\right)$ to $R_{e}$ is given by:

$$
f^{\prime}(\pi) D_{i}(\pi)=-D_{i}(f)(\pi)-A_{i}-B_{i}
$$

in which $f^{\prime}(\pi)$ is the ordinary derivative of $f(x)$ evaluated at $\pi$,

$$
\begin{gathered}
D_{i}(f)(\pi)=p\left[D_{i}\left(a_{2 p-1}\right) \pi^{2 p-1}+D_{i}\left(a_{2 p-2}\right) \pi^{2 p-2}\right. \\
\left.+\cdots+D_{i}\left(a_{1}\right) \pi+D_{i}\left(a_{0}\right)\right] \\
\left\{\begin{array}{c}
A_{i}=p \sum_{k=1}^{2 p-1} S_{k, i}^{*} \text { in which } \\
S_{k, i}^{*}=\sum_{\substack{i_{1}+i_{2}+\cdots+i_{k+1}=i \\
0 \leq i_{j}<i \text { for } j=1,2, \cdots, k+1}}^{*} D_{i_{1}}\left(a_{k}\right) D_{i_{2}}(\pi) \cdots D_{i_{k+1}}(\pi)
\end{array}\right.
\end{gathered}
$$


and

$$
B_{i}=\sum_{\substack{i_{1}+i_{2}+\cdots+i_{2 p}=i \\ 0 \leqq i_{j}<i \text { for } j=1,2, \cdots, 2 p}} D_{i_{1}}(\pi) D_{i_{2}}(\pi) \cdots D_{i_{2 p}}(\pi)
$$

We now prove a lemma which gives sufficient conditions for extending $D^{\prime} \in \mathscr{H}_{c}\left(R, R_{2 p}\right)$ to a $D \in \mathscr{H}_{c}\left(R_{2 p}, R_{2 p}\right)$.

LEMMA 1.1. Let $D^{\prime} \in \mathscr{K}_{c}\left(R, R_{2 p}\right)$ where $R_{2 p}=R[\pi]$ and (1.1) is the minimum polynomial of $\pi$. If for $q>2$, there exist integers $n>1$ and $m>2 p(n-1)$ such that

$$
\begin{gathered}
D_{j}(\pi) \in M(2) \text { for } 0<j<n \\
\left\{\begin{array}{l}
D_{j}(\pi) \in M(q) \text { for } n \leqq j<2 n-1 \\
D_{j}(\pi) \in M(q+1) \text { for } 2 n-1 \leqq j<m
\end{array}\right. \\
D_{j}\left(a_{k}\right) \in M\left(q+1-2 p-k+V\left(f^{\prime}(\pi)\right)\right) \text { for } j \geqq 2 n-1
\end{gathered}
$$

and $k=1,2, \cdots, 2 p-1$ and when $j \geqq m$ for $k=0$ also, then unless $p=3, q=3$, and $V\left(f^{\prime}(\pi)\right)=4 p-1, \sum D_{j}(\pi)$ converges and $\sum_{j=2 n-1}^{\infty} D_{j}(\pi) \epsilon$ $M(q+1)$.

Proof. First we show by induction that $D_{j}(\pi) \in M(q+1)$ if $j \geqq$ $m$. Thus assume $D_{j}(\pi) \in M(p+1)$ for all $j$ such that $2 n-1<j<r$ where $r \geqq m>2 p(n-1)$. From (1.10) it is immediate that $D_{r}(f)(\pi) \in$ $M\left(q+1+V\left(f^{\prime}(\pi)\right)\right)$ since $p \in M(2 p)$. Considering $B_{r}$, observe that $i_{1}+i_{2}+\cdots+i_{2 p}=r>2 p(n-1)$ implying that at least one index in $\left\{i_{1}, i_{2}, \cdots, i_{2 p}\right\}$ is greater than $n-1$ and at least one other is $\neq 0$. Thus each such term is in $M(q+2 p)$. Moreover, each such term appears a multiple of $p$ times unless each distinct index appears a multiple of $p$ times so that the sum of these nonexceptional terms is in $M(4 p+q)$. Thus $V\left(f^{\prime}(\pi)\right) \leqq 4 p-1$ implies that this sum is in $M\left(V\left(f^{\prime}(\pi)\right)+q+1\right)$. In the exceptional case there are three possibilities:

(1) $i_{1}=1_{2}=\cdots=i_{2 p}=(r / 2 p)>(2 p(n-1) / 2 p)=n-1$.

(2) $i_{1}=\cdots=i_{p}$ and $i_{p+1}=\cdots=i_{2 p}$ (relabeling subscripts if necessary) where $i_{1} \neq i_{p+1}$, and $i_{1}, i_{p+1} \neq 0$.

(3) $i_{1}=\cdots=i_{p}=(r / p)>(2 p(n-1) / p)=2(n-1)$ and $i_{p+1}=$ $\cdots=i_{2 p}=0$ (again relabeling subscripts if necessary).

Using (1.9), one checks that the terms in each of these cases are in $M\left(V\left(f^{\prime}(\pi)\right)+q+1\right)$. It follows that $B_{r} \in M\left(V\left(f^{\prime}(\pi)\right)+q+1\right)$. Now considering $A_{r}$, it is straightforward to check the values of the terms to verify that $\left.p S_{k, r}^{*} \in M\left(V^{\prime}(\pi)\right)+q+1\right)$ except when $s=p$ and $i_{1}<2 n-1$. This case will follow if we can show that $p S_{p, r}^{*} \in$ $M\left(V\left(f^{\prime}(\pi)\right)+q+1\right)$. Thus recall $S_{p, r}^{*}$ is a sum of terms of the form 
$D_{i_{1}}\left(a_{p}\right) D_{i_{2}}(\pi) \cdots D_{i_{p+1}}(\pi)$. Now fix $i_{1}$ and observe that for a given set of indices $\left\{i_{1}, i_{2}, \cdots, i_{p+1}\right\}$, the sum of terms with this set of indices is a multiple of $p$ unless $i_{2}=i_{3}=\cdots=i_{p+1}$. In the nonexceptional case at least one index is greater than $n$ so that the sum of these terms together with the coefficient of $p^{2}$ is in $M(5 p+q)$. When $i_{2}=i_{3}=\cdots=i_{p+1}$, each index is greater than $n$ so that each of these terms together with the coefficient $p$ is in $M(p q+2 p)$. It follows that $p S_{p, r}^{*} \epsilon$ $M(p+q) \subset M\left(V\left(f^{\prime}(\pi)\right)+q+1\right)$ since $q>2$ and $p>2$. Thus for each $k, p S_{k, r}^{*} \in M\left(V\left(f^{\prime}(\pi)\right)+q+1\right)$ so that $A_{r} \in M\left(V\left(f^{\prime}(\pi)\right)+q+1\right)$. It follows that $D_{r}(\pi) \in M(q+1)$. It remains to show the convergence of $\sum_{i=0}^{\infty} D_{i}(\pi)$. Thus, given $i>1$ assume for some integer $r \geqq m$ that $j>r$ implies $D_{j}(\pi) \in M(q+i)$. Since $D$ converges on $R$, it is clear that this $r$ may chosen so that $D_{j}\left(a_{k}\right) \in M\left(V\left(f^{\prime}(\pi)\right)+i+q-2 p+1\right)$. Now letting $r^{\prime}=2 p r$, one may check in a manner similar to that given above, that for $j>r^{\prime} D_{j}(\pi) \in M(q+i+1)$. It follows that $\sum D_{j}(\pi)$ converges and $\sum_{j=2 n-1}^{\infty} D_{j}(\pi) \in M(q+1)$.

LEMMA 1.2. If $a_{0} \in f(x)$ is such that $\bar{a}_{0} \in h^{p}$, then for every positive integer $n$ there exists a prime element $\pi_{n}$ for which

$$
\pi_{n}^{2 p}+p\left(v^{p^{n}}+\pi_{n}^{s} w_{n}\right)=0
$$

for some units $v$ and $w_{n}$ in $R_{2 p}$.

Proof. For given $n$ we multiply

$$
\pi^{2 p}+p\left(v^{p}+\pi^{s} w\right)=0
$$

through by $v^{p^{n}-p}$. Letting $\pi_{n}=\pi v^{\left(p^{n-1}-1\right) / 2}$ and $w_{n}$ be the product of $w$ and the remaining factors of $v$, the result follows.

Suppose now that $\alpha \in H_{i} \mid G_{i+1}$ so that $\alpha=e+\pi^{i} \alpha^{*}$, where $e$ is the identity map and $\alpha^{*}$ is an additive mapping on $R_{2 p}$ for which $\alpha^{*}(M) \subset M$. Then the mapping $\phi_{i}(\alpha)$ induced on $h$ by $\alpha^{*}$ is a derivation on $h$. The mapping $\phi_{i}: H_{i} \rightarrow \mathscr{D}(h)$ is a homomorphism with kernel $G_{i+1}$ and for a given $\alpha \in H_{i} \backslash G_{i+1}, \phi_{i}(\alpha)$ will hereinafter be denoted by $\delta_{\alpha}$. Now suppose $\alpha(\pi)=\pi\left(1+\pi^{i} z\right)$ for some $z \in R_{2 p}$. Apply $\alpha$ to (1.2) to obtain

$$
\begin{aligned}
& \pi^{2 p} \sum_{\substack{k=1 \\
k \neq p}}^{2 p}\left(\begin{array}{c}
2 p \\
k
\end{array}\right) \pi^{k i} z^{k}+\pi^{2 p}\left(\begin{array}{c}
2 p \\
p
\end{array}\right) \pi^{p i} z^{p} \\
& \quad+p\left[\pi^{i} \alpha^{*}(u)+\pi^{s+i} \alpha^{*}(w)+\pi^{s} w \sum_{k=1}^{s}\left(\begin{array}{c}
s \\
k
\end{array}\right) \pi^{k i} z^{k}\right. \\
& \left.\quad+\pi^{s+i} \alpha^{*}(w) \sum_{k=1}^{s}\left(\begin{array}{c}
s \\
k
\end{array}\right) \pi^{k i} z\right]=0 .
\end{aligned}
$$


Apply $\alpha$ to (1.3) to obtan

$(1.13, \mathrm{~s})$

$$
\begin{aligned}
& \pi^{2 p} \sum_{\substack{k=1 \\
k \neq p}}^{2 p}\left(\begin{array}{c}
2 p \\
k
\end{array}\right) \pi^{k i} z^{k}+\pi^{2 p}\left(\begin{array}{c}
2 p \\
p
\end{array}\right) \pi^{p i} z^{p} \\
& +p\left[\sum_{k=1}^{p}\left(\begin{array}{l}
p \\
k
\end{array}\right) v^{p-k} \pi^{k i} \alpha^{*}(v)^{k}+\pi^{s+i} \alpha^{*}(w)\right. \\
& \left.+\pi^{s} w \sum_{k=1}^{s}\left(\begin{array}{l}
s \\
k
\end{array}\right) \pi^{k i} z^{k}+\pi^{s+i} \alpha^{*}(w) \sum_{k=1}^{s}\left(\begin{array}{l}
s \\
k
\end{array}\right) \pi^{k i} z^{k}\right]=0 .
\end{aligned}
$$

Lemma 1.3. If $\alpha \in H_{i} \backslash G_{i+1}$ for $i=1,2, \cdots$, then $\delta_{\alpha}(\bar{u})=0$, where $u$ is as in (1.2).

Proof. Consideration of the values of the terms in $(1.11, \mathrm{~s})$ shows that $\alpha^{*}(u) \in M$ since otherwise $p \pi^{i} \alpha^{*}(u)$ would have unique minimum value in $(1.11, \mathrm{~s})$. Thus $\delta_{\alpha}(\bar{u})=0$.

II. Proof of Theorem 1. It is known that for each $i, \phi_{i}\left(H_{i}\right)$ is a subgroup of the additive group of derivations on $h$, and we have seen in Lemma 1.3 that if $\delta \in \phi_{i}\left(H_{i}\right)$, then $\delta(\bar{u})=\delta\left(\bar{a}_{0}\right)=0$. It will be sufficient then to show that we can find an automorphism in $H_{i} \backslash G_{i+1}$ that will induce the desired derivation on $h$. We do this by considering several cases.

Case 1. $i \geqq 2$ and $0<s<p$. Suppose $\delta \in \mathscr{D}_{0}(h)$. It is known [2, Theorem 1], that $\delta$ lifts to a $d \in \mathscr{D}(R)$ for which $d\left(a_{0}\right) \in M(2 p)$ so define a higher derivation $E=\left\{E_{i}\right\}_{i=0}^{\infty} \in \mathscr{C}(R, R)$ as follows: For $j=$ $1,2, \cdots, p-1$, define $E_{j}=d^{j} / j$ ! and for $j \geqq p$, by Theorem A there exist maps $E_{j}$ such that $E=\left\{E_{j}\right\} \in \mathscr{H}(R, R)$.

We want to show that we can construct an $\alpha_{D} \in H_{i} \backslash G_{i+1}$ for $i \geqq 2$ which will induce the given $\delta \in \mathscr{D}(h)$. Thus, define $D_{j}=\pi^{i j} E_{j}$. Clearly, $D=\left\{D_{j}\right\} \in \mathscr{H}_{u}\left(R, R_{2 p}\right)$. All that remains is to show that $D(\pi)$ converges. From (1.5) one sees that for $j=1,2, \cdots, p-1, D_{j}(f)(\pi) \epsilon$ $M(2 p+s+i j)$, and for $j>p-1, D_{j}(f)(\pi) \in M(2 p+i j)$, and it follows that for $j>1, D_{j}(f)(\pi) / f^{\prime}(\pi) \in M(i+2)$. The rest of this case will be concerned then, with the convergence of $\left(A_{j}+B_{j}\right) / f^{\prime}(\pi)$. In considering $A_{j}$ we will usually be concerned with the value of $S_{s, j}^{*}$ since in most cases the term of minimum value will occur in $S_{s, j}^{*}$. For $j=1, A_{1}=0, B_{1}=0$, and $D_{1}(\pi) \in M(i+1) \subset M(3)$, since $i \geqq 2$. Now for $r<j<p$ we suppose that $D_{r}(\pi) \in M(i r+1)$, and consider $D_{j}(\pi)$. Inspection of (1.6) reveals that $S_{s, j}^{*} \in M(i j+s)$ so that $A_{j} \in M(2 p+$ $i j+s)$ and $A_{j} / f^{\prime}(\pi) \in M(i j+1)$. Since $j<p$, each term in $B_{j}$ appears a multiple of $p$ times so that inspection of (1.7) reveals that $B_{j} \in$ $M(4 p+i j)$, and thus $B_{i} / f^{\prime}(\pi) \in M(i j+1)$. Hence, for $j=1,2, \cdots$, 
$p-1, D_{j}(\pi) \in M(i j+1)$. For $j=p, A_{p} \in M(2 p+i p+s)$ by the same analysis as used before so that $A_{p} / f^{\prime}(\pi) \in M(i p+1) \subset M(i+2)$. In $B_{p}$, the term $D_{1}(\pi)^{p} \pi^{p}$ does not occur a multiple of $p$ times, and this is the only term which may not be in $(4 p+i p)$. But $D_{1}(\pi) \in M(i+1)$ so that $D_{1}(\pi)^{p} \pi^{p} \in M(p i+2 p)$ which implies $B_{p} \in M(p i+2 p)$. Thus $B_{p} / f^{\prime}(\pi) \in M(p i-p+2)$ so that $D_{p}(\pi) \in M(i+2)$. Now suppose that for $p<r<j<2 p, D_{r}(\pi) \in M(i+2)$. Then one checks that $A_{j} / f^{\prime}(\pi) \in$ $M(i+2)$. Each term in $B_{j}$ again appears a multiple of $p$ times so that $B_{j} / f^{\prime}(\pi) \in M(i+2)$. Thus $D_{j}(\pi) \in M(i+2)$ for $p<j<2 p$.

In $A_{2 p}$, observe that $S_{s, 2 p}^{*} \in M(s+2 i+2)$, implying that $A_{2 p} / f^{\prime}(\pi) \in$ $M(i+2)$. In $B_{2 p}$ the terms of minimum value are $D_{1}(\pi)^{2 p} \in M(2 p i+2 p)$ and $D_{2}(\pi)^{p} \pi^{p} \in M(2 p i+2 p)$; all other terms in $B_{2 p}$ appear a multiple of $p$ times and it follows that $B_{2 p} \in M(2 p i+2 p)$ and $B_{2 p} / f^{\prime}(\pi) \in M(i+2)$. Thus $D_{2 p}(\pi) \in M(i+2)$.

From the definition of $D, D_{j}(R) \subset M(i j)$. For $0<k<s, D_{j}\left(a_{k}\right) \in$ $M(2 p+i j) \subset f^{\prime}(\pi) M(i+2-2 p-k)$ for $j \geqq 3$. If $k \geqq s, D_{j}\left(a_{k}\right) \in$ $M(i j) \subset M\left(V\left(f^{\prime}(\pi)\right)+i+2-2 p-k\right)$. Thus the hypotheses of Lemma 1.1 are satisfied for $q=i+1, n=2$, and $m=2 p+1$, and $D=\left\{D_{i}\right\}_{i=0}^{\infty}$ converges on $R_{2 p}$. Moreover, $\alpha_{D}=\sum D_{i}$ induces $\delta \in \mathscr{D}_{0}(h)$ since by the construction $D_{1}=\pi^{i} d$ and $D_{j}\left(R_{2 p}\right) \subset M(i+2)$ for $j>1$. It follows that for $0<s<p$ and $i \geqq 2, H_{i} / G_{i+1} \cong \mathscr{D}_{0}(h)$.

Case 2. $i \geqq 3$ and $p<s<2 p$. In this case $D=\left\{D_{i}\right\}$ is constructed exactly as in Case 1. The hypotheses of Lemma 1.1 are satisfied for $q=i+1, n=2$ and $m=2 p+1$ since conditions (1.10) are the same as in Case 1, and it follows that in this case $H_{i} / G_{\imath+1} \cong \mathscr{D}_{0}(h)$.

Case 3. $i=2$ and $p<s<2 p-2, p \neq 3$. Again construct $D$ as in Case 1, and apply Lemma 1.1 using $q=3, n=2$, and $m=2 p+1$ to see that $D(\pi)$ converges. Thus for $p<s<2 p-2, p \neq 3, H_{2} / G_{3} \cong$ $\mathscr{D}_{0}(h)$.

Case 4. $i=1$ and $s<p-1$. Assume first that prime element $\pi$ has been chosen so that if $\bar{u} \in h^{p}$, the $v$ of (1.3) is a $p$ th power. Then $a_{0}=c_{0}^{p^{2}}+p c_{1}$ for some units $c_{0}$ and $c_{1}$ in $R$. If $\bar{u} \notin h^{p}$, then choose $\bar{S}$, a $p$-basis for $k$, so that $\bar{u} \in \bar{S}$. Now let $\delta \in \mathscr{D}_{0}(h)$ and suppose that $\delta$ lifts to $d \in \mathscr{D}(R)$. Since $\delta \in \mathscr{D}_{0}(h), d\left(a_{0}\right) \in M(2 p)$. For $j=$ $1,2, \cdots, p-1$ let $D_{j}=\pi^{j} d^{j} / j$ ! so all the results in Case 1 hold for these values of $j$, i.e., for $i=1, D_{j}(\pi) \in M(j+1)$ for $0<j<p$. At this point we separate into several subcases.

Case 4(i). $s<p-2, p \neq 3$. If $\bar{S}$ is a $p$-basis for $h$, let $S$ be a set of representatives in $R$ of $\bar{S}$. Then for $j \geqq p$ define $D_{j}$ by letting $D_{j}(S)=0$ which implies that $D_{i}(R) \subset M(j)$ for $j \geqq p$ by Lemma A. 
One then checks that the hypotheses of Lemma 1.1 are satisfied when $q=3, n=2$, and $m=2 p+1$. Thus $D=\left\{D_{i}\right\}$ converges and $\alpha_{D}=$ $\sum_{i=0}^{\infty} D_{i}$ is in $H_{1}$ and induces $\delta \in \mathscr{D}_{0}(h)$.

Case 4(ii). $s=p-2, i=1$, and $\bar{w} \in k^{p}$. In this case observe first that for a given $\delta \in \mathscr{D}_{0}(h), \delta(\bar{w})=0$. Then lifting $\delta$ to $d \in \mathscr{D}(R)$, $d\left(a_{0}\right) \in M(2 p)$, and $d\left(a_{s}\right) \in M(2 p)$. Defining $D_{j}=\pi^{j} d^{j} / j$ ! for $j=1,2$, $\cdots, p-1$ as before, $D_{j}(\pi) \in M(j+2)$. For $j \geqq p$ define $D_{j}$ by letting $D_{j}(S)=0$. Then $D_{j}(R) \subset M(j)$, and observe that $D_{j}(f)(\pi) \in M(2 p+s+j)$ for $j \geqq p$, i.e. if $\bar{a}_{0} \notin h^{p}$, then $D_{j}\left(a_{0}\right)=0$ for every $j \geqq p$, and if $\bar{a}_{0} \in h^{p}$, then $a_{0}=c_{0}^{p 2}+p c_{1}$ from the first remarks so that $D_{j}\left(a_{0}\right) \in$ $M(2 p+1)$. In either case $D_{j}(f)(\pi) \in M(2 p+s+j)$. It follows that for all $j \geqq p, D_{j}(f)(\pi) / f^{\prime}(\pi) \in M(j+1)$. In arguments that are routine by now, $B_{p} \in M(4 p)$ so that $B_{p} / f^{\prime}(\pi) \in M(4)$, and $A_{p} / f^{\prime}(\pi) \in M(4)$. Standard arguments also show that $D_{j}(\pi) \in M(4)$ for $p<j \leqq 2 p$ so that $D(\pi)$ converges by Lemma 1.1 when $q=3, n=2$, and $m=2 p+1$.

Case 4(iii). $s=p-2, i=1, \bar{w} \notin h^{p}$, and $\bar{u} \in h^{p}$. The construction for a given $\delta \in \mathscr{D}_{0}(h)$ which lifts to $d \in \mathscr{D}(R)$ is the same as before for $j=1,2, \cdots, p-1$, i.e., $D_{j}=\pi^{j} d^{j} / j$ ! for these values of $j$, so that $D_{j}(\pi) \in M(j+1)$. Continuing, $A_{p} \in M(3 p+s)$ so that $A_{p} / f^{\prime}(\pi) \in M(p+1)$. $B_{p}$ contains the term $2 D_{1}(\pi)^{p} \pi^{p}$ so the fact that $D_{1}(\pi) \in M(2)$ implies that $B_{p} \in M(3 p)$. Choosing $S$ so that $\bar{a}_{p-2}=\bar{w} \in \bar{S}$ define $D_{p}$ to be such that $p D_{p}\left(\bar{a}_{p-2}\right) \pi^{p-2}+B_{p} \in M(3 p+1)$ and define $D_{p}\left(S \backslash a_{p-2}\right)=0$. Then $D_{p}(f)(\pi)+B_{p}+A_{p} \in M(3 p+1)$ so that $D_{p}(\pi) \in M(4)$. For $j>p$ define $D_{j}(S)=0$ so that if $j=p m+k, 0 \leqq k<p$, then from Lemma A, $D_{j}(R) \subset M(2 m+k)$. We want to show that $D_{j}(\pi) \in M(4)$ for all $j>p$. We do this by an induction. Thus suppose that $D_{r}(\pi) \in M(4)$ for all $r$ such that $2<r<t$. Then, $D_{t}(f)(\pi) \in M(2 p+s+4)$ so that $D_{t}(f)(\pi) / f^{\prime}(\pi) \in M(5)$. Next $A_{t} \in M(2 p+s+4)$ so that $A_{t} / f^{\prime}(\pi) \in M(5)$. In showing that $B_{t} / f^{\prime}(\pi) \in M(4)$ we need to consider two cases: $p \nmid t$, (2) $t=m p, m \geqq 2$. In case (1) each term of $B_{t}$ occurs a multiple of $p$ times so that $B_{t} \in M(4 p+4)$ and $B_{t} / f^{\prime}(\pi) \in M(p+6) \subset M(4)$. In case (2) all terms appear a multiple of $p$ times except for the following three cases:

(i ) $i_{1}=i_{2}=\cdots=i_{p}=(m p / p) ; i_{p+1}=\cdots=i_{2 p}=0$.

(ii) $i_{1}=i_{2}=\cdots=i_{2 p}=(m p / 2 p)$ so $m=2 \ell$ for some $\ell \geqq 1$.

(iii) $i_{1}=i_{2}=\cdots=i_{p}=r_{1} ; i_{p+1}=\cdots=i_{2 p}=r_{2}$ where $r_{1}+r_{2}=$ $m, r_{1} \neq r_{2}$, and $r_{1}, r_{2} \neq 0$.

In (i), $D_{m}(\pi)^{p} \pi^{p} \in M(4 p)$; in (ii), $D_{l}(\pi)^{2 p} \in M(4 p)$; and in (iii), $D_{r_{1}}(\pi)^{p} D_{r_{2}}(\pi)^{p} \in M(5 p)$. Thus $B_{m p} \in M(4 p)$, and

$$
B_{m p} / f^{\prime}(\pi) \in M(4 p-3 p+3)=M(p+3) .
$$

Therefore $D_{t}(\pi) \in M(4)$. We now apply Lemma 1.1, taking $q=3$ and 
$n$ and $m$ sufficiently large so that the hypotheses of the lemma are satisfied.

Case 4(iv). $p-2 \leqq s<p$ or $p<s<2 p, i=1$, and $\bar{u} \notin h^{p}$. Again we begin the construction with a $\delta \in \mathscr{D}_{0}(h)$ so that $\delta$ that $\delta$ lifts to a $d \in \mathscr{D}(R)$ for which $d\left(a_{0}\right) \in M(2 p)$, and define $D_{j}=\pi^{j} d^{j} / j$ ! for $0<j<p$. From the previous case this implies that $D_{j}(\pi) \in M(j+1)$ for $0<$ $j<p$. As before, $A_{p} \in M(3 p+s)$ so that $A_{p} / f^{\prime}(\pi) \in M(p+1)$. $B_{p}$ contains $2 D_{1}(\pi)^{p} \pi^{p} \in M(3 p)$ so choose $S$ so that $a_{0} \in S$ and define $D_{p}$ to be such that $p D_{p}\left(a_{0}\right)+B_{p} \in M(2+s+3)$, and define $D_{p}\left(S \backslash a_{0}\right)=0$. Then $D_{p}(R) \subset M(p)$ and $D_{p}(\pi) \in M(4)$. For $p<j<2 p$ define $D_{j}(S)=0$. Routine calculation shows that $D_{j}(\pi) \in M(4)$ for $p<j<2 p$. $B_{p} \in$ $M(4 p)$ since it contains $D_{1}(\pi)^{2 p} \in M(4 p)$ and $D_{2}(\pi)^{p} \pi^{p} \in M(4 p)$. If $s<2 p-2$, then define $D_{j}(S)=0$ for all $j \geqq p$. If $s=2 p-2$ or $2 p-1$, define $D_{2 p}\left(a_{0}\right)$ so that $p D_{p}\left(a_{0}\right)+B_{p} \in M(2 p+s+3)$, and $D_{2 p}\left(S \backslash a_{0}\right)=0$. For $j>2 p$ define $D_{j}(S)=0$. In either case $D_{j}(R) \subset$ $M(j)$, and $A_{2 p} / f^{\prime}(\pi) \in M(5)$. Also $D_{2 p}(f)(\pi) \in M(4 p+s)$ so that $D_{2 p}(\pi) \in$ $M(4)$. Thus apply Lemma 1.1 , letting $q=3, n=2$, and $m=2 p+1$. It follows that in all of the subcases considered in Case $4, H_{1} / G_{2} \cong$ $\mathscr{D}_{0}(h)$.

Case 5. $s=p-1, i=1, \bar{u} \in h^{p}$ and $\bar{w} \notin h^{p}$. Recall that $v$ in equation (1.3) is such that $\bar{v} \in h^{p}$, so that for any $\delta \in \mathscr{D}(h), \delta(\bar{v})=0$. Thus in equation $(1.13, p-1), \alpha^{*}(v) \in M$, so that pulling out terms of minimum value and simplifying, we have

$$
2 v^{p} z^{p}+w z-\alpha^{*}(w)=0 \quad \bmod \pi .
$$

Thus any $\delta \in \dot{\phi}_{1}\left(H_{1}\right)$ is such that

$$
\delta(\bar{w})=\rho\left(2 v^{p} z^{p}+w z\right)
$$

for some $\bar{z} \in h$.

Since $\bar{w} \notin h^{p}$ choose a $p$-basis $\bar{S}$ for $h$ which contains $\bar{a}_{p-1}=\bar{w}$ and choose $S$ to contain $a_{p-1}$. Let $\delta \in \mathscr{D}(h)$ be any derivation such that $\delta(\bar{w})=\rho\left(2 v^{p} a^{p}+w a\right)$. We want to show that there exists an $\alpha_{D} \in H_{1}$ which induces $\delta$. So suppose $\delta$ lifts to $d^{\prime} \in \mathscr{D}(R)$. Then define $d \in R$ by setting $d(\xi)=d^{\prime}(\xi)$ for every $\xi \in S \backslash\left\{a_{p-1}\right\}$ and setting $d\left(a_{p-1}\right)=a_{p-1}$ $a$ where $a$ is a representative for some given $\bar{a} \in h$. Let $D_{1}=\pi d$. Observation of (1.4) shows that $D_{1}(\pi)=\pi^{2} a \bmod \pi^{3}$. For $j=2, \cdots$, $p-1$ define $D_{j}(S)=0$. Then $D_{j}(R) \subset M(j)$ and $D_{j}(\pi) \in M(j+1)$ for $j=1,2, \cdots, p-1$. In checking the $p$ th map in $D=\left\{D_{j}\right\}$ observe that $S_{p, p-1}^{*} \in M(s+p)$ so that $A_{p} \in M(3 p+s)$ and $A_{p} / f^{\prime}(\pi) \in M(p+1)$. Also note that every term in $B_{p}$ except the term $D_{1}(\pi)^{p} \pi^{p} \in M(3 p)$ appears a multiple of $p$ times so that except for this term, $B_{p} \in$ 
$M(4 p)$. Now define $D_{p}$ by letting $D_{p}\left(a_{p-1}\right)$ be such that $p \pi^{p-1} D_{p}\left(a_{p-1}\right)+$ $B_{p} \in M(3 p+2)$ and $D_{p}(\xi)=0$ for every $\xi \in S \backslash\left\{a_{p-1}\right\}$. Then $D_{p}(R) \subset M$ and $D_{p}(\pi) \in M(4)$. For $j>p$ define $D_{j}(S)=0$. Observe that if $j=$ $r p+k$, where $0 \leqq k<p$, then by Lemma $\mathrm{A}, D_{j}(R) \subset M(r+k)$ so that $D \in \mathscr{H}_{u}\left(R, R_{2 p}\right)$. To prove that $D(\pi)$ converges we use an induction like that used in the proof of Case 4(iii) to show that for all $j \geqq p$, $D_{j}(\pi) \in M(4)$. Then we apply Lemma 1.1 with $q=3$ and $m$ and $n$ sufficiently large so that conditions (1.9) and (1.10) are satisfied.

Note that in this construction $D_{1}\left(a_{p-1}\right)=a a_{p-1} \pi$ and that in defining $D_{p}$, we choose $D_{p}\left(a_{p-1}\right)$ so that $p \pi^{p-1} D_{p}\left(a_{p-1}\right)+B_{p} \in M(3 p+2)$. The term of least value in $B_{p}$ is $\left(\begin{array}{c}2 p \\ p\end{array}\right) D_{1}(\pi)^{p} \pi^{p}=2 a^{p} \pi^{3 p}$. Thus $p \pi^{p-1} D_{p}(w)+$ $2 \alpha^{p} \pi^{3 p} \in M(3 p+2)$, and since $p \equiv-v^{-p} \pi^{2 p}(\bmod M(3 p-1)), D_{p}\left(a_{p-1}\right) \equiv$ $2 a^{p} v^{p} \pi \bmod \pi^{2}$. For $j \neq 1, p D_{j}\left(a_{-p_{1}}\right)=0$ by definition, so that for $\alpha_{D}=\sum_{j=0}^{\infty} D_{j}, \rho\left(\alpha_{D}^{*}\left(a_{p-1}\right)\right)=\rho\left(a w+2 a^{p} v^{p}\right)$, and $\alpha_{D}$ induces $\delta$.

Case 6. $s=p-1, i=1, \bar{u} \in h^{p}$, and $\bar{w} \in h^{p}$. This case is the same as the previous one except that $\bar{a}_{p-1}=\bar{w} \in h^{p}$. Thus for every $\delta \in \mathscr{D}(h), \delta(\bar{w})=0$, so suppose $\delta \in \mathscr{D}(h) . \quad \delta$ lifts to a $d \in \mathscr{D}(R)$ such that $d\left(a_{9}\right) \in M(2 p)$ and $d\left(a_{p-1}\right) \in M(2 p)$. For $j=1,2, \cdots, p-1$ define $D_{j}=\pi^{j} d^{j} / j$ ! and for $j>p-1$ define $D_{j}(S)=0$. It is straightforward to verify that $D=\left\{D_{j}\right\} \in \mathscr{H}_{c}\left(R_{2 p} R_{2 p}\right)$ and that $\alpha_{D}=\sum_{j=0}^{\infty} D_{j} \in$ $H_{1} \mid G_{2}$. It follows that every $\delta \in \mathscr{D}(h)$ is induced by an $\alpha \in H_{1}$ so that $H_{1} / G_{2} \cong \mathscr{D}(h)$.

Case 7. $p<s<2 p-2, p \neq 3, i=1, \bar{u} \in h^{p}$. Observation of $(1.13, \mathrm{~s})$ reveals that since $\bar{v} \in h^{p}, \alpha^{*}(w) \in M$. It follows then that if $\delta \in \phi_{1}\left(H_{1}\right)$, $\delta(\bar{w})=\delta\left(\bar{a}_{p-1}\right)=0$, or $\phi_{1}\left(H_{1}\right) \subset \mathscr{D}_{1}(h)$.

Now let $\delta \in \mathscr{D}_{1}(h)$. Lifting $\delta$ to $d \in \mathscr{D}(R)$ and letting $D_{j}=\pi^{j} d^{j} / j$ ! for $j=1,2, \cdots, p-1, D_{j}(R) \subset M(j)$, and $D_{j}(\pi) \in M(j+2)$. For $j \geqq p$ define $D_{j}$ by letting $D_{j}(S)=0$, and it follows by Lemma A that $D_{j}(R) \subset M(j)$ for all values of $j$. The argument that $D(\pi)$ converges is standard and will be omitted, except to note that $D_{j}(\pi) \in M(4)$ for $2<j \leqq 2 p$ so we can show convergence using Lemma 1.1 with $q=3$, $n=2$, and $m=2 p+1$. It follows that the $\alpha_{D}=\sum D_{i}$ obtained induces the given $\delta \in \mathscr{D}_{1}(h)$ so that $H_{1} / G_{2} \cong \mathscr{D}_{1}(h)$ in this case.

Case 8. $s=2 p-2, i=1$, and $\bar{u} \in h^{p}$. As before, assume that for $\alpha \in H_{1} \mid G_{2}, \alpha(\pi)=\pi(1+\pi z)$ and that $\bar{v} \in h^{p}$. It follows that $\alpha^{*}(v) \in$ $M$. Further, consideration of the values of the terms in $(1.13,2 p-2)$ reveals that $\alpha^{*}(v) \in M$ implies that $z \in M$. It follows that $\alpha^{*}(w) \in M$ and, as a result, if $\delta \in \phi_{1}\left(H_{1}\right)$, then $\delta(\bar{w})=0$. We will show that $\phi_{1}\left(H_{1}\right) \cong \mathscr{D}_{1}(h)$, (recalling that if $\bar{w} \in h^{p}, \mathscr{D}_{1}(h)=\mathscr{D}(h)$ ) by constructing a derivation automorphism that induces $\delta$. Thus suppose $\delta \in \mathscr{D}_{1}(h)$. 
Lifting $\delta$ to $d \in \mathscr{D}(R)$ and letting $D_{j}=\pi^{j} d^{j / j !}$ for $j=1,2, \cdots, p-1$ one may check that $D_{j}(R) \subset M(j)$ and $D_{j}(\pi) \in M(j+2)$. At this point we separate into several subcases.

Case 8(i). If $\bar{w} \notin h^{p}$, we choose a $p$-basis $\bar{S}$ for $h$ to contain $\bar{w}$. Letting $S$ be a set of representatives of $\bar{S}$, define $D_{p}$ by letting $D_{p}(w)$ be such that $D_{p}(\pi) \in M(4 p+1)$ and $D_{p}(S \backslash\{w\})=0$. For $j>p$ define $D_{j}$ by letting $D_{j}(S)=0$. Observe that $D_{p}(R) \subset M(2)$ so that by Lemma A $D=\left\{D_{i}\right\} \in \mathscr{K}_{c}\left(R, R_{2 p}\right)$. One may then check that $D(\pi)$ converges using Lemma 1.1, with $n=(p+3) / 2, m=p^{2}+p$, and $q=3$.

Case 8(ii). $\quad \bar{w}_{0} \in h^{p}$ and $\bar{w}_{1} \in h^{p}$ where we specialize (1.3) by writing

$$
\pi^{2 p}+p\left(v^{p}+\pi^{2 p-2} w_{0}+\pi^{2 p-1} w_{1}\right)=0 .
$$

Note that $\bar{w}_{0}=\bar{w}=\bar{a}_{2 p-2}$ and $\bar{w}_{1}=\bar{a}_{2 p-1}$. One may check that in this case $D_{j}(\pi) \in M(j+3)$ for $j=1,2, \cdots, p-1$. For $j \geqq p$, define $D_{j}(S)=$ 0 . It is routine to verify by standard arguments that $D=\left\{D_{j}\right\}$ converges in this case.

Case 8(iii). $\quad \bar{w}_{0} \in h^{p}$ and $\bar{w}_{1} \notin h^{p}$. We choose a $p$-basis for $h$ to include $\bar{w}_{1}$ and define $D_{p}$ by letting $D_{p}(\bar{w})$ be such that $D_{p}(\pi) \in M(4)$ and $D_{p}(S \backslash\{w\})=0$. For $j>p$, define $D_{j}$ by $D_{j}(S)=0$. Observe that by Lemma A, $D_{k p}(R) \subset M(k)$ and for $j=k p+\ell, 0<\ell<p, D_{j}(R) \subset$ $M(k+\ell)$. Thus $D \in \mathscr{C}_{c}\left(R, R_{2 p}\right)$. Using Lemma 1.1, it is routine to verify that $D=\left\{D_{i}\right\}$ converges by taking $q=3, n=2 p$, and $m=$ $2 p(2 p-1)+1$.

Case 9(i). $s=2 p-1, i=1, \bar{u} \in h^{p}$, and $\bar{w} \notin h^{p}$. From $(1.13,2 p-1)$ it is apparent that $z$ is not a unit. Thus letting $z=\pi y$, the fact that minimum value terms must be congruent implies that $\alpha^{*}(w) \equiv$ $2^{p} v^{p} y^{p}(\bmod \pi)$. To show that $\phi_{1}\left(H_{1}\right) \cong \mathscr{D}_{4}(h)$, it will suffice to show that we can construct an $\alpha_{D} \in H_{1} \mid G_{2}$ for which $\phi_{1}\left(\alpha_{D}\right)(\bar{w})=\rho\left(2^{p} v^{p} b^{p}\right)$ for any given $\bar{b}^{p} \in h^{p}$. Thus let $\delta \in \mathscr{D}(h)$ be such that $\delta(\bar{w})=0$. We assume that $a_{2 p-1} \in S$ so that $\bar{a}_{2 p-1}=\bar{w} \in \bar{S}$. Then $\delta$ lifts to a $d^{\prime} \in$ $\mathscr{D}(R)$ and we define $d \in \mathscr{D}(R)$ by letting $d(\xi)=d^{\prime}(\xi)$ for all $\xi \in S \backslash\left\{a_{2 p-1}\right\}$, and we define $d\left(a_{2 p-1}\right)=\pi^{2} b a_{2 p-1}$.

Thus the derivation in $\mathscr{D}(h)$ induced by $d$ and $d^{\prime}$ agree on $\bar{S}$ so they are equal. For $j=1,2, \cdots, p-1$ define $D_{j}=\pi^{j} d^{j} / j$ ! , and one may verify that $D_{i}(R) \subset M(j)$ and $D_{j}(\pi) \in M(j+2)$. In particular $D_{1}(\pi) \equiv \pi^{3} b\left(\bmod \pi^{4}\right)$. Now note that $B_{p}$ contains the term $2 b^{p} \pi^{4 p}$ and that all other terms in $A_{p}$ and $B_{p}$ have higher value. Thus define $D_{p}\left(a_{2 p-1}\right)$ to be such that $D_{p}(f)(\pi)+B_{p} \in M(4 p+2)$ and define $D_{p}\left(S \backslash a_{2 p-1}\right)=0 . \quad$ It follows that $D_{p}\left(a_{2 p-1}\right) \equiv 2^{p} b^{p} v^{p} \pi(\bmod M(2)), D_{p}(\pi) \epsilon$ $M(4)$, and $D_{p}(R) \subset M$. 
For $j>p$ define $D_{j}$ by $D_{j}(S)=0$. Then if $j=m p+k, 0 \leqq k<$ $p, D_{j}(R) \subset M(m+k)$. This clearly converges on $R_{2 p}$ by an induction argument similar to that used in Case 4(iii) and Lemma 1.1. Moreover, $\alpha_{D}^{*}\left(a_{2 p-1}\right) \equiv a^{p} b^{p} v^{p}(\bmod \pi)$ so that $\alpha_{D}$ induces a derivation of the desired kind. It follows that $H_{1} / G_{2} \cong \mathscr{D}_{4}(h)$ in this case.

Case 9(ii). Same as 9(i) except $\bar{a}_{2 p-1} \in h^{p}$. In this case $\pi$ satisfied equation (1.4) in which $\bar{w}_{0} \in h^{p}$. Applying $\alpha$ to (1.4) and considering values as before, $\delta_{\alpha}(\bar{w})=0$, i.e., $\delta_{\alpha} \in \mathscr{D}_{5}(h)$. Recalling that $a_{0}=c_{0}+p c_{1}$, $\delta \in \mathscr{D}_{5}(h)$ implies that $\delta\left(\bar{c}_{1}\right)=0$. Lifting $\delta$ to a $d \in \mathscr{D}(R)$, observe that since $\bar{w}_{1}=\bar{c}_{1}$, and $\bar{v} \in h^{p}, d\left(a_{0}\right) \in M(4 p)$, and since $\bar{a}_{2 p-1} \in h^{p}$, $d\left(a_{2 p-1}\right) \in M(2 p)$. Thus letting $D_{j}=\pi^{j} d^{j} / j$ ! for $j=1,2, \cdots, p-1$, $D_{j}(\pi) \in M(j+3)$ and $D_{j}(R) \subset M(j)$. For $j \geqq p$ define $D_{j}(S)=0$ so that $D_{j}(R) \subset M(j)$ for all $j$ and $D=\left\{D_{j}\right\} \in \mathscr{K}_{u}\left(R, R_{2 p}\right)$. The standard arguments show that $D(\pi)$ also converges so that $\alpha_{D}=\sum_{j=0}^{\infty} D_{j}$ is an automorphism in $H_{1} \mid G_{2}$ which induces the given $\delta$. It follows that $H_{1} / G_{2} \cong \mathscr{D}_{5}(h)$.

Case 10. $i=2, s=2 p-2$, and $\bar{u} \in h^{p}$. This case is analogous to Cases 5 and 6 . Note that the terms of minimum value in (1.13, $2 p-2)$ when $i=2$ are such that

$$
\rho\left(2 v^{p} z^{p}+2 w z-\alpha^{*}(w)\right)=0 .
$$

Thus if $\delta \in \phi_{2}\left(H_{2}\right)$, then

$$
\delta(\bar{w})=\rho\left(2 v^{p} a^{p}+2 w a\right)
$$

for some $\bar{a} \in h$. The analysis from here on is exactly analogous to that of $H_{1} / G_{2}$ when $s=p-1$ except that we replace $\mathscr{D}_{2}(h)$ with $\mathscr{D}_{3}(h)$, i,e., if $\bar{w} \notin h^{p}$ then $H_{2} / G_{3} \cong \mathscr{D}_{3}(h)$, and if $\bar{w} \in h^{p}$, then $H_{2} / G_{3} \cong$ $\mathscr{D}(h)$.

Case 11. $i=2, s=2 p-1$, and $\bar{u} \in h^{p}$. Observation of the values of successive terms in $(1.13,2 p-1)$ when $i=2$ reveals that $z$ cannot be a unit. Moreover, since $\bar{v} \in h^{p}, \alpha^{*}(w) \in M$. Thus $\dot{\phi}_{2}: H_{2} \rightarrow \mathscr{D}_{1}(h)$. As usual we show that $\phi_{2}$ is surjective by constructing a higher derivation automorphism.

Let $\delta \in \mathscr{D}_{1}(h)$ so that $\delta(\bar{w})=\delta\left(\bar{a}_{2 p-1}\right)=0$. This $\delta$ lifts to $d \epsilon$ $\mathscr{D}(R)$, and, as before, define $D_{j}=\pi^{2 j} d^{j} / j$ ! for $j=1,2, \cdots, p-1$. Then the usual calculation shows that $D_{j}(R) \subset M(2 j)$ and $D_{j}(\pi) \in$ $M(2 j+2)$ for these values. This means that $D_{1}(\pi) \in M(4)$ so the minimum value term in $B_{p}, D_{1}(\pi)^{p} \pi^{p}$, is in $M(5 p)$. For $j \geqq p$, we define $D_{j}$ by letting $D_{j}(S)=0$ and the usual calculation shows that $D$ is a convergent higher derivation. It follows that $\dot{\phi}_{2}: H_{2} \rightarrow \mathscr{D}_{1}(h)$ is surjective and that in this case $H_{2} / G_{3} \cong \mathscr{D}_{1}(h)$, 
Case 12. $i=2, s=2 p-2$ or $2 p-1$, and $\bar{u} \notin h^{p}$. As before, assume that $\bar{u}=\bar{a}_{0} \in \bar{S}$. Let $\delta \in \mathscr{D}(h)$ be such that $\delta\left(\bar{a}_{0}\right)=0 . \quad \delta$ lifts to a $d \in \mathscr{D}(R)$ for which $d\left(a_{0}\right) \in M(2 p)$. Define $D_{j}=\pi^{2 j} d^{j} / j$ ! for $j=$ $1,2, \cdots, p-1$. Then $D_{j}(R) \subset M(2 j)$ and $D_{j}(\pi) \in M(2 j+1)$ for these values of $j$. Observe that $B_{p}$ contains the term $D_{1}(\pi)^{p} \pi^{p} \in M(4 p)$ which does not appear a multiple of $p$ times. Thus define $D_{p}$ by letting $D_{p}\left(a_{0}\right)$ be such that $p D_{p}\left(a_{0}\right)+B_{p} \in M(2 p+s+3)$, and define $D_{p}\left(S \backslash a_{0}\right)=$ 0 . Then $D_{p}(R) \subset M(p)$ and routine calculation shows that $D_{p}(\pi) \in$ $M(4)$. Now for $j>p$, define $D_{j}(S)=0$, and by standard arguments, $D=\left\{D_{j}\right\}$ converges. Thus in this case $H_{2} / G_{3} \cong \mathscr{D}_{0}(h)$.

To complete the proof of the theorem we need to consider the ramification groups that occur when $s=p$ and $s=2 p$. One may verify that they are obtained by same procedures as have been used in the previous cases. Only two deserve special mention.

Case 13. $s=2 p, i=3, \bar{u} \in h^{p}, \bar{w} \notin h^{p}$, and $p=3$. In this case routine calculation reveals that for any $\alpha \in H_{3} \mid G_{4} \delta_{\alpha}(\bar{w})=\rho\left(2 v^{p} z-2 v^{2 p} z^{p}\right)$ for some $\bar{z} \in h$. To show that $\phi_{3}\left(H_{3}\right)$ maps onto $\mathscr{D}_{6}(h)$ one uses a construction similar to that used in Case 5.

Case 14. $s=p, \bar{u}, \bar{w} \in h^{p}$. In this case we prove

Lemma 2.1. Suppose $s=p, \bar{u}, \bar{w} \in h^{p}$. Then the factors $H_{i} / G_{i+1}$ are as given when $s=\sigma$ and $a_{\sigma}$ assumes the role of $a_{s}$.

Proof. For the conditions stated $\pi$ has the property that

$$
\pi^{2 p}+p\left(v_{1}^{p}+w_{1}^{p} \pi^{p}+y_{1} \pi^{\sigma}\right)=0
$$

for some units $v_{1}, w_{1}$, and $y_{1}$. Since we are assuming a $p$-basis for $h$ is nonempty we can choose a prime element $\pi$ such that $v_{1} \in R$ and $\bar{v}_{1} \notin h^{p}$. Also, we choose $S$ to include $v_{1}$. We construct an inertial embedding of $R$ into $R_{2 p}$ by defining a higher derivation $D=\left\{D_{j}\right\}$ on $R$ as follows. Let $D_{1}\left(v_{1}\right)=\pi w_{1}, D_{1}\left(S \backslash\left\{v_{1}\right\}\right)=0$, and $D_{j}(S)=0$ for $j>1$. Then $D \in \mathscr{C}_{u}\left(R, R_{2 p}\right)$ and $\beta=\sum_{k=0}^{\infty} D_{k}$ is the desired inertial embedding. Let $R^{\prime}=\beta(R)$ and note that

$$
v_{1}^{p}+w_{1}^{p} \pi^{p} \equiv\left(v_{1}+w_{1} \pi\right)^{p}(\bmod M(2 p)) .
$$

Letting $v=\left(v_{1}+w_{1} \pi\right)$ and $y=y_{1}-\left[\left(v_{1}+\pi w_{1}\right)^{p}-v_{1}^{p}-w_{1}^{p} \pi^{p}\right] / \pi^{\sigma}$ then $\pi^{2 p}+p\left(v^{p}+y \pi^{\sigma}\right)=0$. Then $\pi$ satisfies an Eisenstein polynomial over $R^{\prime}$ of degree $2 p$ in which $s=\sigma$. The conclusion follows.

To complete the proof of the theorem we note that if $\bar{u} \in h^{p}$, $\mathscr{D}_{0}(h)=\mathscr{D}(h)$, and if $\bar{w} \in h^{p}, \mathscr{D}_{1}(h)=\mathscr{D}(h)$. 
III. Galois theory. In this section we characterize wildly ramified normal extensions of degree $q p$, for $q<p$. We let $R_{q}$ denote a tamely ramified extension of degree $q$ so that if $\gamma$ is a prime element for $R_{q}, R_{q}=R[\gamma]$. Moreover, from [12, Theorem 3-4-3] we may choose a $\gamma$ which satisfies the Eisenstein equation

$$
x^{q}+p y=0
$$

in $R[x]$. Further, assume that $\pi$ is a prime element for $R_{p q}$ so that $R_{q p}=R_{q}[\pi]$, and that the minimum polynomial of $R_{p q}$ over $R_{q}$ is

$$
g(x)=x^{p}+\gamma \sum_{i=0}^{p} b_{i} x^{i} .
$$

Also $\Gamma\left(R_{e} / R\right)$ will always denote the Galois group of $R_{e}$ over $R$.

For convenience we state the following well known lemmas:

Lemma 3.1. Let $\alpha \in \Gamma\left(R_{q} / R\right)$. If $\alpha \in H_{i}$, then $\alpha \in G_{i+1}$.

LEMMA 3.2. $\quad R_{q}$ is a Galois extension of $R$ if and only if $h$ contains a primitive $q$ th root of unity. Moreover, if $\alpha \in \Gamma\left(R_{q} / R\right)$, $\alpha(\gamma)=\theta \gamma$ where $\theta$ is a qth root of unity in $R$.

Let $t^{*}$ denote the residue of $t$ modulo $p-1,0 \leqq t^{*}<p-1$, and let [ ] denote the greatest integer function. We restate the theorem from [1] with some notational modifications.

THEOREM 4. Suppose $R_{p q}, R_{q}$, and $R$ are as above; let tp $=$ $\min \left\{V\left(b_{i}\right) \mid i=1,2, \cdots, p-1\right\}$, and let $j$ be the least positive integer $i$ such that $V\left(b_{i}\right)=t p$. If $b_{1}=\cdots=b_{p-1}=0$, set $t=+\infty$ and $j=1$. Then necessary and sufficient condition for $R_{p q} / R_{q}$ to be normal are:

Case 1. $t<q$

(a) $j=p-1-t^{*}$

(b) $\rho\left(-j b_{j} /\left(\gamma^{t}\left(-b_{0}\right)^{t+1}\right)\right)$ has a $(p-1)$ th root.

Case 2. $t \geqq q$

(c) $q=r(p-1), r$ an arbitrary, positive integer, and

(d) $\rho\left(-\gamma^{q} / p\right)$ has a $(p-1)$ th root.

Moreover, the nontrivial Galois automorphisms of $R_{p q} / R_{q}$ are in $G_{n} \backslash H_{n}$ where

$$
n=\{t+2+[t / p-1] \text { in Case 1, rp }+1 \text { in Case } 2\} .
$$

LEMMA 3.3. Let $\alpha \in \Gamma\left(R_{q} / R\right)$ and let $\theta$ and $\xi$ be representatives of $q$ th roots of unity in $h$ such that $\theta^{p} \equiv \xi(\bmod M)$. If $\alpha(\gamma)=\xi \gamma$, then if $\alpha$ extends to $R_{p q}, \alpha(\pi) \equiv \theta \pi(\bmod M(2))$. 
Proof. From Lemma $3.2 \alpha(\gamma)=\xi \gamma$. If $\alpha$ extends to $R_{p q}$, then $\alpha(\pi)=\pi(1+z)$. Also recall that $\alpha \in G_{i}$ for some $i \geqq 1$. But $\pi$ satisfies an equation $\pi^{p}+\gamma u_{1}=0$ for some unit $u_{1}$ so that by applying $\alpha$ to this equation we obtain the equation

$$
\pi^{p} \sum_{k=1}^{p}\left(\begin{array}{l}
p \\
k
\end{array}\right) z^{k}+\gamma u_{1}(\xi-1)+\gamma \pi^{i} \alpha^{*}\left(u_{1}\right)=0 .
$$

Inspection of (3.3) reveals that $z$ must be a unit, for otherwise $\gamma u_{1}(\xi-1)$ would have unique minimum value. It follows that

$$
\pi^{p} z^{p}+\gamma u_{1}(\xi-1) \equiv 0(\bmod M(p+1)) .
$$

Thus $\bar{z}^{p}=\rho(\xi-1)$ so that $z \equiv \theta-1(\bmod \pi)$ and as a result $\alpha(\pi) \equiv$ $\theta \pi(\bmod M(2))$.

It is clear from this lemma that any nontrivial $\alpha^{\prime} \in \Gamma\left(R_{q} / R\right)$ that extends to $R_{p q}$ extends to an $\alpha \in G_{1} \backslash H_{1}$.

We conclude this section on Galois theory with

THeORem 5. Let $f(x)=x^{p q}+p \sum_{i=0}^{p q-1} a_{i} x^{i}$ be the minimum polynomial of $R_{p q} / R$, and let $s$ be as defined in $\S \mathrm{I}$. Then $R_{p q} / R$ is Galois if and only if $h$ contains a primitive $q$ th root of unity and one of the sets of conditions below is satisfied:

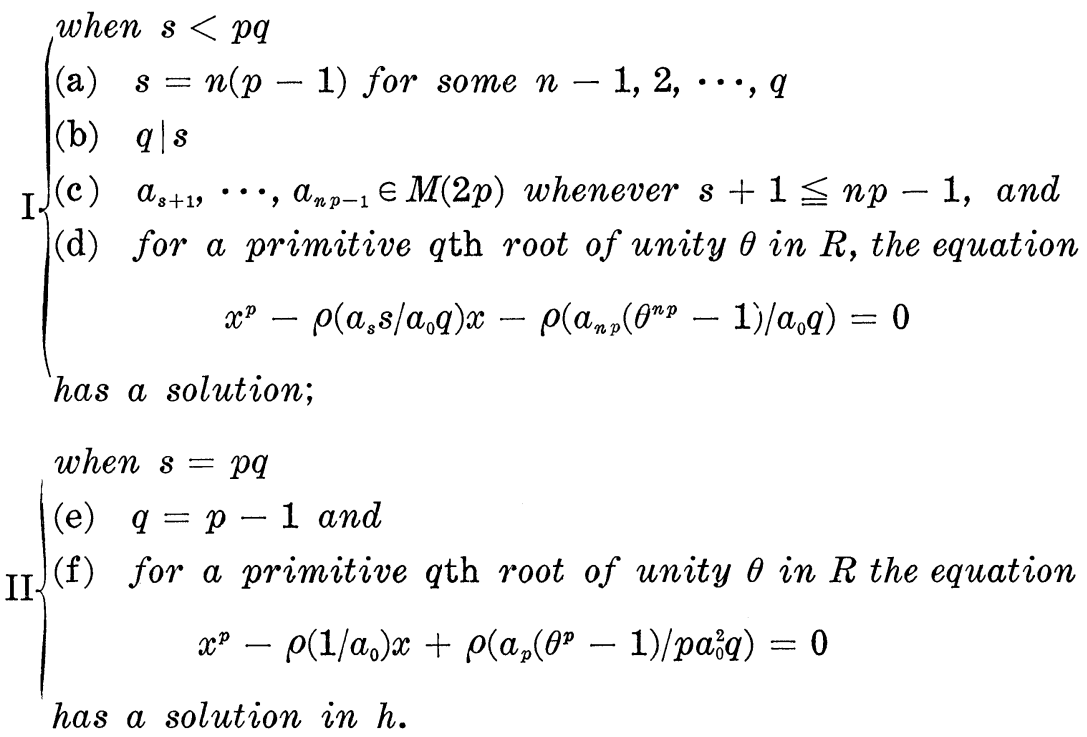

Proof. The method of proof is similar to that of Theorem 4 . Assuming that $R_{p q} / R$ is Galois, we apply an $\alpha \in \Gamma\left(R_{p q} / R\right)$ to $f(\pi)=0$ and observe that the given conditions are necessary. To prove they are sufficient, we use the conditions to construct all the roots of $f(x)$ 
in much the same way as in the proof of Theorem 4. Thus, suppose $R_{p q} / R$ is Galois. Then $R_{q} / R$ is Galois so that by Lemma $3.2 h$ contains the $q$ th roots of unity. Now let $\theta$ be the unique multiplicative representative in $R$ of a primitive $q$ th root of unity $\bar{\theta}$ in $h$. Lemmas 3.2 and 3.3 imply that the action of $\alpha$ on $\pi$ will be of the form $\alpha(\pi)=\pi\left(\theta+z \pi^{n}\right)$ for some unit $z$ and some integer $n>0$. Using the fact that $f(\alpha(\pi))=0$, some straightforward manipulation yields:

$$
\begin{gathered}
\pi^{p q} \sum_{k=1}^{p q}\left(\begin{array}{c}
p q \\
k
\end{array}\right) \theta^{p q-k} z^{k} \pi^{n k}+p \sum_{i=1}^{p q-1} a_{i} \pi^{i}\left(\theta^{i}-1\right) \\
+p \sum_{i=1}^{p q-1} a_{i} \pi^{i} \sum_{k=1}^{i}\left(\begin{array}{c}
i \\
k
\end{array}\right) \theta^{i-k} z^{k} \pi^{n k}=0
\end{gathered}
$$

Suppose first that $s<p q$. Since $R_{p q} / R$ is Galois, the fact that (3.4) holds for a primitive $q$ th root implies that it must hold for every $q$ th root of unity $\theta_{1}$. In particular it must hold when $\theta_{1}=1$ in which case the middle sum vanishes. Since $s<p q$, the value of the last sum is less than $2 p q+n$ so the term of index $k=p$ in the first sum has minimal value. It follows that $s=n(p-1)$ so that (a) is necessary. Note that $s=n(p-1)$ implies that $n<p$. Conversely, if $n<p$, inspection of (3.4) reveals that $s<p q$. Thus for future reference we note that $s<p q$ if and only if $n<p$.

Recalling the definition of $s$ and that (3.4) must hold for every $q$ th root of unity $\theta_{1}, p a_{s} \pi^{s}\left(\theta_{1}^{s}-1\right)$ will be a term of unique minimum value in (3.4) unless $\left(\theta_{i 1}^{s}-1\right) \in M$ for every $q$ th root of unity $\theta_{1}$. Thus $q \mid s$. Similarly $a_{s+1}, \cdots, a_{n p-1} \in M(2 p)$ so (b) and (c) are necessary. Simplification of the residues of the minimum value terms in (3.4) leads to the equation

$$
x^{p}-\rho\left(a_{s} s / a_{0} q\right) x-\rho\left(a_{n p}\left(\theta^{n p}-1\right) / a_{0} q\right)=0
$$

in which $z=\theta x$. Thus (d) is necessary.

Suppose now that $s=p q$. As before the middle term of (3.4) vanishes when $\theta=1$. Equating the values of terms of minimum value yields $n=p q /(p-1)$. It follows that $n=p$ and $q=p-1$ so that (e) is necessary. The equation in $h$ resulting from the fact that the sum of the minimum value terms in (3.4) must be congruent $\bmod M(2 p q+n+1)$ is $(f)$ were $z=\theta x$. Thus conditions II are necessary when $s=p q$.

To prove the sufficiency, we construct the roots by induction in a manner similiar to that used in in the proof of Theorem 4 . If we assume conditions I of theorem and if for a given $q$ th root of unity $\theta$, we let $e_{1}$ be a representative in $R_{p q}$ of a root of (d), then the first approximation for a root of $f(x)$ is $\pi\left(\theta+\pi^{n} e_{1}\right)$, and one may verify 
that $f\left[\pi\left(\theta+\pi^{n} e_{1}\right)\right] \in M(p q+n p+1)$. As before, assume that we have chosen $e_{2}, e_{3}, \cdots, e_{m}$ so that, for $\lambda_{m}=\theta+\pi^{n} e_{1}+\pi^{n+1} e_{2}+\cdots+$ $\pi^{n+m-1} e_{m}, f\left(\pi \lambda_{m}\right) \in M(p q+n p+m)$. We then show that for $\lambda_{m+1}=$ $\lambda_{m}+\pi^{n+m} e_{m+1}$, we can choose an $\mathrm{e}_{m+1}$ for which $f\left(\pi \lambda_{m+1}\right) \in M(p q+n p+$ $m+1)$. Thus by induction for a given primitive $q$ th root of unity $\theta$ we can construct a root of $f(x)$. It follows that we can construct a root of $f(x)$ for every $q$ th root of unity $\theta_{1}$, including $\theta_{1}=1$. Moreover (d) must have a solution for every $q$ th root of unity $\theta_{1}$ so that when $\bar{\theta}_{1}=\overline{1}$, the equation

$$
\bar{x}^{p-1}-\rho\left(a_{s} s / a_{0} q\right)=0
$$

must have a solution. If $\bar{\xi}$ denotes a solution for (3.6) and $\bar{\eta}$ denotes a solution for

$$
x^{p}-\rho\left(a_{s} s / a_{0} q\right) x-\rho\left(a_{n p}\left(\theta_{1}^{n p}-1\right) / a_{0} q\right)=0
$$

for a given $q$ th root of unity $\bar{\theta}_{1}$, then one may verify that a complete set of solutions for $\left(3.7, \theta_{1}\right)$ is given by $\{\bar{\eta}+r \bar{\xi} \mid r=0,1,2, \cdots, p-1\}$. It follows that $R_{p q}$ contains $p$ roots of $f(x)$ for each $q$ th root of unity $\bar{\theta}_{1}$ and from their construction it is clear that each is distinct. Therefore we have constructed $p q$ roots of $f(x)$, so that $R_{p q} / R$ is Galois and conditions (I) are sufficient. Conditions (II) also imply that $R_{p q} / R$ is Galois in much the same manner as conditions (I). The main difference is that the first approximation for a root of $f(x)$ is given by $\pi\left(\theta+e_{1} \pi^{p}\right)$, where $e_{1}$ is a representative of a solution of $(f)$.

IV. Proofs of Theorems 2 and 3. Now that the location of the Galois groups in the ramification sequence has been determined, we can prove Theorems 2 and 3 .

First, for $i>1$ and $\alpha \in G_{i}$, we define $\psi_{i}(\alpha)$ to be the residue in $h$ of $(\alpha(\pi)-\pi) / \pi^{i}$. Then one may verify that $\psi_{i}: G_{i} \rightarrow h$ is a homomorphism of $G_{i}$ into $h^{+}$, the additive group of $h$, with kernel $H_{i}$. With this observation the proof of Theorem 2 follows from a sequence of lemmas.

Lemma 4.1. Let $i>1$ and $\bar{u} \notin h^{p}$. Then for every $\bar{a} \in h^{+}, \bar{a} \neq 0$, there exists an $\alpha \in G_{i} \backslash H_{i}$ for which $\psi_{i}(\alpha)-\bar{a}$.

Proof. Let $a \in R_{2 p}$ be a representative of $\bar{a} \in h$. We prove this as lemma well as several of the following ones by constructing a convergent higher derivation $D=\left\{D_{j}\right\}$ such that $D_{1}(\pi)=\pi^{i} a$ and $D_{j}(\pi) \epsilon$ $M(i+1)$ for all $j>1$. Then for $\alpha_{D}=\sum D_{i}, \psi_{i}\left(\alpha_{D}\right)=\bar{a}$. Thus let $\bar{S}$ be a $p$-basis for $h$ that includes $\bar{u}=\bar{a}_{0}$, and choose $S \subset R_{2 p}$ to be a set of representatives of $\bar{S}$ such that $a_{0} \in S$. Suppose now that 
$s \neq p$ and define $D_{1}\left(a_{0}\right)=-f^{\prime}(\pi) \pi^{i} a / p$ and $D_{i}\left(S \backslash\left\{a_{0}\right\}\right)=0$. For $j=$ $2, \cdots, p-1$, define $D_{j}(S)=0$. Then $D_{1}(R) \subset M\left(V\left(f^{\prime}(\pi)\right)+i-2 p\right)$ and by Lemma A $D_{j}(R) \subset M\left(V\left(f^{\prime}(\pi)^{j}\right)+i j-2 p j\right)$. Moreover $D_{1}(\pi)=$ $\pi^{i} a$ and $D_{j}(\pi) \in M(i j+1)$. At this point we separate into several cases.

Case 1. $s \neq p$ and $i=2$. The term of minimum value in $A_{p}+B_{p}$ is $2 D_{1}(\pi)^{p} \pi^{p} \in M(3 p)$. If $s \leqq p-3$, define $D_{j}(S)=0$ for $j \geqq p$. If $p-3<s \leqq 2 p$, define $D_{p}\left(S \backslash\left\{a_{0}\right\}\right)=0$ and $D_{p}\left(a_{0}\right)$ such that $D_{p}(f)(\pi)+$ $A_{p}+B_{p} \in M(2 p+s+3)$. Then in either case, $D_{p}(R) \subset M(p)$ and $D_{p}(\pi) \in M(4)$. Define $D_{j}(S)=0$ for $p<j<2 p$ and if $p-3<s<p$ or $p<s<p-2$ define $D_{j}(S)=0$ for $j \geqq 2 p$. Since the term of minimum value in $A_{2 p}+B_{2 p}$ is $D_{1}(\pi)^{2 p} \in M(4 p)$, for $2 p-2 \leqq s \leqq 2 p$, define $D_{2 p}\left(S \backslash\left\{a_{0}\right\}\right)=0$ and $D_{2 p}\left(a_{0}\right)$ such that $D_{2 p}(f)(\pi)+A_{2 p}+B_{2 p} \epsilon$ $M(2 p+s+4)$, and for $j>2 p$ define $D_{j}(S)=0$. Now observe that $D_{2 p}(R) \subset M(2 p)$ so that if $j=m p+r$ for $0 \leqq r<p$, from Lemma A, $D_{j}(R) \subset M(m p+2 r)$. Thus $D=\left\{D_{j}\right\} \in \mathscr{H}_{c}\left(R, R_{2 p}\right)$, and it is routine to verify that $D_{j}(\pi) \in M(5)$ for $p<j \leqq 2 p$ so that Lemma 1.1 implies convergence of $D$ by taking $q=3, n=2$, and $m=2 p+1$.

Case 2. $s \neq p$ and $i>2$. Again the term of minimum value in $A_{p}+B_{p}$ is $2 D_{1}(\pi)^{p} \pi^{p} \in M(p i+p)$. If $0<s<2 p-2$, define $D_{j}(S)=0$ for $j \geqq p$. Then $D_{j}(R) \subset M(j i)$ and $D_{j}(\pi) \in M(i+1)$ for $p \leqq j \leqq 2 p$. Thus $D$ converges by Lemma 1.1 by taking $q=i, n=2$, and $m=$ $2 p+1$. If $2 p-2 \leqq s \leqq 2 p$, define $D_{p}\left(S \backslash\left\{a_{0}\right\}\right)=0$ and $D_{p}\left(a_{0}\right)$ such that $D_{p}(f)(\pi)+A_{p}+B_{p} \in M(2 p+s+i)$. Then $D_{p}(R) \subset M(p)$ and $D_{p}(\pi) \in$ $M(i+1)$. For $j>p$ define $D_{j}(S)=0$ so that for $j=m p+r$ for $0 \leqq r<p, D_{j}(R) \subset M(m p+r i)$. Clearly $D=\left\{D_{j}\right\} \in \mathscr{H}_{c}\left(R, R_{2 p}\right)$ and for $p<j \leqq 2 p, D_{j}(\pi) \in M(i+1)$. Then $D(\pi)$ converges by Lemma 1.1, taking $q=i, n=2$, and $m=2 p+1$.

Case 3. $i>1$ and $s=p$. Let $\sigma$ be the least positive integer greater than $p$ for which $a_{\sigma}$ is a unit, or if $a_{p+1}, \cdots, a_{2 p-1} \in M(2 p)$, let $\sigma=2 p$. Then the construction of the convergent higher derivation is the same as in the previous two cases if we let $\sigma$ assume the role of $s$. To prove this we need to show that the fact that $s=p$ does not interfere with the convergence of those constructions. To do this, first observe that if $\bar{a}_{p} \in h^{p}$, then we can choose a prime element such that $a_{p}=1+p c_{p}$ for some $c_{p} \in R$, i.e., $\bar{a}_{p} \in k^{p}$ implies that $a_{p}=$ $d_{1}^{p}+p d_{2}$ for some $d_{1}, d_{2} \in R$. Then let $\pi^{\prime}=\pi d_{1}^{-1}$ be a new prime element so that if $x^{2 p}+p \sum_{i=0}^{2 p-1} a_{i}^{\prime} x^{i}$ is the minimum polynomial of $\pi^{\prime}$, then $a_{p}^{\prime}=1+p c_{p}$ where $c_{p}=d_{2} d_{1}^{-p}$, and $\rho\left(a_{0} d^{-2 p}\right) \notin h^{p}$. Thus we assume that we have chosen a prime element of this form so that $a_{p}=1+p c_{p}$ for some $c_{p}$. It follows that for every $j, V\left(D_{j}\left(a_{0}\right)\right)<$ 
$V\left(\pi^{p} D_{j}(a)\right)$. If $\bar{a}_{p} \notin h^{p}$ and $\bar{a}_{0}$ and $\bar{a}_{p}$ are $p$-dependent, it is clear that for every $j, V\left(D_{j}\left(a_{0}\right)\right)<V\left(\pi^{p} D_{j}\left(a_{p}\right)\right)$. If $\bar{a}_{p} \notin h^{p}$, and $a_{0}$ and $a_{p}$ are $p$-independent, then we choose $S$ to include $a_{p}$ as well as $a_{0}$. From our construction, then $D_{j}\left(a_{p}\right)=0$ for every $j$. Thus $V\left(D_{j}\left(a_{0}\right)\right)<$ $V\left(\pi^{p} D_{j}\left(a_{p}\right)\right)$ so that in any of these cases $V\left(D_{j}(f)(\pi)\right)$ is independent of $V\left(D_{j}\left(a_{p}\right)\right)$, and the fact that $s=p$ does not interfere with the convergence of the constructions.

In all of these constructions, for a given $i>1$, we have a $D=$ $\left\{D_{j}\right\}$ such that $D_{1}(\pi)=\pi^{i} a$ and $D_{j}(\pi) \in M(i+1)$ for $j>1$. It follows that for $\alpha_{D}=\sum D_{j}, \psi_{i}\left(\alpha_{D}\right)=\bar{a}$. Thus $\alpha_{D} \in G_{i} \backslash H_{i}$. But from the construction $\alpha_{D}(a)-a \in M(i)$ for every $a \in R$, so that $\alpha_{D} \in G_{i} \backslash H_{i}$, completing the proof of the lemma.

LEMMA 4.2. Let $i>1$ and $\bar{u} \notin h^{p}$. For $\psi_{i}$ as described above, $\psi_{i}\left(G_{i}\right) \cong G_{i} / H_{i} \cong h^{+}$.

Proof. As noted above, for a given prime element $\pi, \psi_{i}: G_{i} \rightarrow h^{+}$ is clearly a homomorphism into $h^{+}$with kernel $H_{i}$. By Lemma 4.1, $\psi_{i}: G_{i} \rightarrow h^{+}$is surjective. Thus $\psi_{i}\left(G_{i}\right)=h^{+}$and $G_{i} / H_{i} \cong h^{+}$.

Before going further we need to observe a few facts about the relationship between (1.1) and (3.2). Let $b_{i}=b_{0 i}+b_{1 i} \gamma$ with $b_{0 i}, b_{1 i} \in R$. Then denoting the conjugate of $a \in R_{2}$ by $\operatorname{conj}(a), \operatorname{conj}\left(b_{i}\right)=b_{0 i}-\gamma b_{1 i}$, and letting conj $(g(x))$ denote the conjugate polynomial of $g(x), f(x)=$ $g(x)(\operatorname{conj} g(x))$. Then by using $\gamma^{2}=-p y$ from (3.1) one may obtain the following relationships among the coefficients:

$$
a_{0}=y b_{00}^{2}+p y^{2} b_{10}^{2}
$$

$$
\begin{aligned}
& a_{i}=y \sum_{j+k=i} b_{0 j} b_{0 k}+y^{2} p \sum_{j+k=i} b_{1 j} b_{1 k} \text { for } 0<i<p \\
& a_{i}=-2 y b_{1 m}+y \sum_{\substack{j+k=i \\
j, k \neq p}} b_{0 j} b_{0 k}+y^{2} p \sum_{\substack{j+k=i \\
j, k \neq p}} b_{1 j} b_{1 k} \\
& \quad \text { for } \quad p \leqq i=m+p<2 p \text { and } m=0,1, \cdots, p-1 .
\end{aligned}
$$

With these notation conventions we prove

LemmA 4.3. Let $t=\min \left\{V\left(b_{i}\right) / p \mid i=1,2, \cdots, p-1\right\}$ and let $j$ be the least positive integer such that $V\left(b_{j}\right)=t p$. Then

(i) $0<s<p$ if and only if $t=0$ if and only if $j=s$.

(ii) $s=p$ if and only if $t \geqq 1$ and $b_{10}$ is a unit.

(iii) $p \leqq s<2 p$ if and only if $t=1$ if and only if $j=m$ where $s=p+m$.

Proof. Proving (i) first, observe from (4.1) that for $0<s<p$ 
$(4.1, \mathrm{~s})$

$$
a_{s}=2 y b_{00} b_{0 s}+y \sum_{\substack{n+k=s \\ n, k \neq s}} b_{0 n} b_{0 k}+y^{2} p \sum_{m+k=s} b_{1 m} b_{1 k} .
$$

It follows that $t=0$, since if all of the $b_{0 i}, i=1,2, \cdots, p-1$ were nonunits, $a_{s}$ would be a nonunit. Conversely, if $t=0, b_{0 j}$ is a unit, which implies that $a_{j}$ is a unit. Thus $s \leqq j$ by definition of $s$ and it follows that $0<s<p$ since $0<j<p$. Moreover, if $s<j,(4.1, \mathrm{~s})$ for $0<s<p$ shows $a_{s}$ would not be a unit. Thus $j=s$, and the proof of (i) is complete when we observe that if $j=s$, then $0<s<p$ by definition of $j$.

Statement (ii) follows from (i) and the equation

$$
a_{p}=-2 y b_{10}+y \sum_{\substack{n+k=p \\ n, k \neq p}} b_{0 n} b_{0 k}+y^{2} p \sum_{\substack{n+k=p \\ n, k \neq p}} b_{1 n} b_{1 k} .
$$

To prove (iii) we consider for $p<s<2 p$

$$
a_{s}=-2 y b_{1 m}+y \sum_{\substack{n+k=s \\ n, k \neq p}} b_{0 n} b_{0 k}+y^{2} p \sum_{\substack{n+k=8 \\ n, k \neq p}} b_{1 n} b_{1 k}
$$

and observe that $0<m<p$. Thus if $p<s<2 p, b_{1 m}$ is a unit since (i) implies that for $p<s<2 p$, the $b_{0 i}, i=1,2, \cdots, p-1$, are all nonunits. Conversely, if $t=1, b_{1 j}$ is a unit, which implies that $a_{p+j}$ is a unit. Arguing as before, (iii) follows.

Suppose now that $\bar{u}=\bar{a}_{0} \in h^{p}$. Then $\pi$ has the property $\pi^{2 p}+$ $p\left(v^{p}+w \pi^{s}\right)=0$ for some $v, w \in R_{2 p}$, and suppose that $s \neq p$. If $\alpha \in$ $G_{i} \backslash H_{i}, \alpha(\pi)=\pi+\pi^{i} z$ for some unit $z$. Applying $\alpha$ to the above equation and simplifying, we obtain

$$
\begin{aligned}
\pi^{2 p} \sum_{k=1}^{2 p}\left(\begin{array}{c}
2 p \\
k
\end{array}\right) \pi^{k(i-1)} z^{k} & +p\left[\sum_{k=1}^{p}\left(\begin{array}{l}
p \\
k
\end{array}\right) v^{p-k} \pi^{k i} \alpha^{*}(v)^{k}+\pi^{s+i} \alpha^{*}(w)\right. \\
& \left.+\left(w+\pi^{i} \alpha^{*}(w)\right) \pi^{s} \sum_{k=1}^{s}\left(\begin{array}{l}
s \\
k
\end{array}\right) \pi^{k(i-1)} z^{k}\right]=0 .
\end{aligned}
$$

LEMMA 4.4. If $i>1, \bar{a}_{0} \in h^{p}$, and $s \neq p$, then $G_{i} \neq H_{i}$ if and only is $R_{2 p} / R_{2}$ if Galois, and in this case $G_{i} / H_{i}$ is the group of order $p$.

Proof. Considering successive values for $i$ in (4.2) and equating the values of the terms of minimum value, we have the following cases:

$i=2$. Then $2 p+s+1=3 p$ implying that $s=p-1$. Moreover, the terms of minimum value are congruent $\bmod M(3 p+1)$ and since $\bar{a}_{0}=\bar{v}^{p}, \bar{a}_{p-1}=\bar{w}$ and $\pi^{2 p} \equiv-p a_{0}(\bmod M(2 p+1))$ this congruence implies that

$$
\bar{z}^{p-1}=\rho\left(a_{p-1}(p-1) / 2 a_{0}\right)
$$


Thus $\alpha \in G_{2} \backslash H_{2}$ implies that $\rho\left(a_{p-1}(p-1) / a_{0}\right)$ has a $(p-1)$ th root. By Lemma 4.3, $s=p-1$ implies that $t=0$ and $j=p-1$. Also substituting for the $a_{i}$ from $(4.1, \mathrm{i})$, and observing that $\bar{b}_{0 p-1}=\bar{b}_{p-1}$ and $\bar{b}_{00}=\bar{b}_{0}$, we have that

$$
\rho\left(a_{p-1}(p-1) / 2 a_{0}\right)=\rho\left(\left(b_{p-1}(p-1) / b_{0}\right)\right)
$$

so that conditions (a) and (b) of Theorem 4 are satisfied. It follows that $\alpha$ is a Galois map.

$i=3$. Then $2 p+s+2=4 p$ so that $s=2 p-2$. It follows from Lemma 4.3 that $t=1$ and $j=p-2$. The minimum value terms are congruent $\bmod M(4 p+1)$ which leads to

$$
\bar{z}^{p-1}=\rho\left(a_{2 p-2}(2 p-2) / 2 a_{0}\right),
$$

implying that $\rho\left(a_{2 p-2}(2 p-2) / 2 a_{0}\right)$ has a $(p-1)$ th root. Observe now, that $t=1$ and $j=p-2$ imply that $b_{p-2}=p c+\gamma b_{1, p-2}$ for some $c \in R$ and where $b_{1, p-2}$ is a unit. Thus $\bar{b}_{1, p-2}=\rho\left(b_{p-2} / \gamma\right)$. It follows from $(4.1, \mathrm{i})$ that

$$
\rho\left(a_{2 p-2}(2 p-2) / 2 a_{0}\right)=\rho\left(-b_{p-2}(p-2) / \gamma\left(-b_{0}\right)^{2}\right)
$$

has a $(p-1)$ th root so that (a) and (b) of Theorem 4 are satisfied. Thus if $\alpha \in G_{3} \backslash H_{3}, \alpha$ is a Galois map.

$i=4$. Then $p=3$ and $s=2 p$ which means that $a_{i} \in M(2 p)$ for $i=1,2, \cdots, 2 p-1$. Also, as before, this implies that $\rho\left(a_{0} z^{2}-1\right)=$ $\overline{0}$ or that

$$
\bar{z}^{2}=\rho\left(1 / a_{0}\right) .
$$

But $\rho\left(a_{0}\right)=\rho\left(y b_{0}^{2}\right)$ so that (4.3) implies that $\bar{y}$ has a square root. It follows that we have the second case of Theorem 4 and that $\alpha$ is a Galois automorphism.

$i>4$. Then $G_{i}=H_{i}$ since otherwise $\pi^{2 p}\left(\begin{array}{c}2 p \\ 1\end{array}\right) \pi^{i-1} z$ would have unique minimum value in (4.2). Thus every $\alpha \in G_{i} \backslash H_{i}$ is a Galois automorphism, and if $G_{i} \neq H_{i}, G_{i} / H_{i}$ has order $p$.

LEMMA 4.5. Let $i>1, \bar{a}_{0} \in h^{p}$ and $s=p$, and suppose $\bar{a}_{p} \in h^{p}$. Then $G_{i} \neq H_{i}$ if and only if $R_{2 p} / R_{2}$ is Galois. If $R_{2 p} / R_{2}$ is Galois, then $G_{i} / H_{i}$ has order $p$.

Proof. Recall from the discussion in Case 3 of the proof of Lemma 4.1, that in the case under construction, we can choose the 
prime element so that $\bar{a}_{p}=1+p c_{p}$. Assuming this, then $\pi$ has the property

$$
\pi^{2 p}+p\left(v^{p}+\pi^{p}+\pi^{o} w\right)=0,
$$

for some units $v$ and $w$ in $R_{2 p}$. In case $\sigma=2 p$, we alter $w$ to obtain

$$
\pi^{2 p}+p\left(v^{p}+\pi^{p}+p w^{\prime}\right)=0,
$$

for units $v$ and $w^{\prime}$ in $R_{2 p}$. Now let $\alpha \in G_{i} \backslash H_{i}$ so that $\alpha(\pi)=\pi+\pi^{i} z$ for some unit $z$. Applying $\alpha$ to (4.4) and simplifying, we obtain

$$
\begin{aligned}
\pi^{2 p} \sum_{k=1}^{2 p}\left(\begin{array}{c}
2 p \\
k
\end{array}\right) \pi^{k(i-1)} \boldsymbol{z}^{k}+p\left[\sum_{k=1}^{p}\left(\begin{array}{l}
p \\
k
\end{array}\right) v^{p-k} \pi^{k i} \alpha^{*}(z)\right. \\
+\pi^{p} \sum_{k=1}^{p}\left(\begin{array}{l}
p \\
k
\end{array}\right) \pi^{k(i-1)} \boldsymbol{z}^{k}+\pi^{\sigma+i} \alpha^{*}(w) \\
\left.+\left(w+\pi^{i} \alpha^{*}(w)\right) \pi^{\sigma} \sum_{k=1}^{o}\left(\begin{array}{l}
\sigma \\
k
\end{array}\right) \pi^{k(i-1)} \boldsymbol{z}^{k}\right]=0 .
\end{aligned}
$$

In an analysis similar to that of Lemma 4.4, one finds that $i=2$ implies that $G_{2}=H_{2}$ and that $i=3$ implies that $\sigma=2 p-2$ and that $\bar{z}^{p-1}=\rho\left(w \sigma / 2 v^{p}\right)$. But $\bar{w}=\bar{a}_{\sigma}, \bar{v}^{p}=\bar{a}_{0}$ and $\bar{\sigma}=-\overline{2}$. Thus from the relations $(4.1,2 p-2)$ and Lemma 4.3 it follows that $\rho\left(-b_{p-2}(p-2) /\left(\gamma\left(-b_{0}\right)^{2}\right)\right.$ has a $(p-1)$ th root and that $j=p-2$ so that the first set of conditions of Theorem 4 is satisfied since $V\left(b_{p-2} / \gamma\right)=0$. When $i=4$ we find that $\sigma=2 p$ and $p=3$, so applying $\alpha$ to (4.5) and using the fact that the minimum value terms must be in $M(3 p+1)$, it follows that

$$
\bar{z}^{p-1}=\rho\left(1 / y b_{0}^{2}\right)
$$

so that $p=3$ implies that $\bar{y}$ has a square root. Thus the second set of conditions of Theorem 4 is satisfied. The same sort of analysis shows that if $i>4, G_{i}=H_{i}$. Therefore, in this case if $G_{i} \neq H_{i}, R_{2 p} / R_{2}$ is Galois and $G_{i} / H_{i}$ is the group of order $p$. The converse follows from the fact that the Galois maps are always in $G_{i} \backslash H_{i}$ for some $i$.

Lemma 4.6. Let $i>1, \bar{a}_{0} \in h^{p}, s=p$, and $\bar{a}_{p} \notin h^{p}$. Then $G_{2}=H_{2}$ and $\psi_{i}\left(G_{i}\right) \cong G_{i} / H_{i} \cong h^{+}$for $i>2$.

Proof. For $\alpha \in G_{2} \backslash H_{2}$, one finds that applying $\alpha$ to (1.3) with $s=p$ yields an equation having a term of unique minimum value which is impossible. Thus $G_{2}=H_{2}$.

Suppose now that $i>2$ and $\sigma$ is as defined in Case 3 of the proof of Lemma 4.1. Then one can verify that $V\left(f^{\prime}(\pi)\right)=2 p+\sigma-1$. Also, we choose $S$ to include $a_{p} . \quad \psi_{i}: G_{i} \rightarrow h^{+}$is a homomorphism with kerne 
$H_{i}$, so we show here that $\psi_{i}$ is surjective. Let $\bar{a} \in h^{+}$and let $a \in R_{2 p}$ be a representative of $a$. Then define $D_{1}\left(a_{p}\right)=-\left(f^{\prime}(\pi) \pi^{i-p} a\right) / p$, and $D_{1}\left(S \backslash\left\{a_{p}\right\}\right)=0$. Then $D_{1}(\pi) \equiv \pi^{i} a(\bmod M(i+1))$ and $D_{1}(R) \subset M(\sigma+i-p)$. For $i<j<p$ define $D_{j}(S)=0$ so that $D_{j}(R) \subset M(i j+j)$, and $D_{j}(\pi) \in$ $M(i j+j+1)$. At this point we separate into cases.

Case 1. $i>3$ or $i=3, p<\sigma<2 p-3$, and $p \neq 3$. For $j \geqq p$, define $D_{j}(S)=0$. Then $D_{j}(R) \subset M(i j+j)$. The term of minimum value in $A_{p}+B_{p}$ is $2 D_{1}(\pi)^{p} \pi^{p} \in M(i p+p)$ so that $D_{p}(\pi) \in M(i+2)$. For $p<j \leqq 2 p$, one can verify that $D_{j}(\pi) \in M(i+2)$. Thus $D(\pi)$ converges by Lemma 1.1 taking $q=i+1, n=2$, and $m=2 p+1$.

Case 2. $i=3$ and $2 p-2 \leqq \sigma \leqq 2 p$. Define $D_{p}\left(a_{p}\right)$ so that $D_{p}(f)(\pi)+A_{p}+B_{p} \in M(2 p+t+4)$. Then $D_{p}(R) \subset M(p)$ and $D_{p}(\pi) \epsilon$ $M(5)$. For $j>p$ define $D_{j}(S)=0$, so that for $j=m p+r, 0 \leqq r<p$, $D_{j}(R) \subset M(m p+r j+r)$ by Lemma A and $D \in \mathscr{L}_{u}\left(R, R_{2 p}\right)$. Also $D_{j}(\pi) \in M(5)$ for $p<j \leqq 2 p$. Thus $D(\pi)$ converges by Lemma 1.1 taking $q=4, n=2$, and $m=2 p+1$.

In both cases for a given $i, \psi_{i}\left(\alpha_{D}\right)=\bar{\alpha}$ and $\alpha_{D} \in G_{i} \backslash H_{i}$. Thus $\psi_{i}: G_{i} \rightarrow h^{+}$is surjective and it follows that $\psi_{i}\left(G_{i}\right) \cong G_{i} / H_{i} \cong h^{+}$.

The rest of this section is concerned with the factor $G_{1} / H_{1}$.

LEMmA 4.7. If $G_{1} \neq H_{1}$, then $G_{1} / H_{1}$ is isomorphic to the group of order 2.

Proof. Let $\alpha \in G_{1} \backslash H_{1}$ and suppose $\alpha(\pi)=\pi+\pi z$ for some unit $z$. Observe that $\pi$ satisfies an equation $\pi^{2 p}+p u=0$ for some unit $u \in R_{2 p}$, and applyinu $\alpha$ to it, we obtain

$$
\pi^{2 p} \sum_{k=1}^{2 p}\left(\begin{array}{c}
2 p \\
k
\end{array}\right) z^{p}-\pi \alpha^{*}(u) p=0
$$

Inspection of this equation reveals that the terms of minimum value are $\pi^{2 p}\left(\begin{array}{c}2 p \\ p\end{array}\right) z^{p}$ and $\pi^{2 p} z^{2 p}$. Their sum must be in $M(2 p+1)$ which implies that

$$
\rho\left(z^{2 p}+2 z^{p}\right)=\overline{0} .
$$

Thus $\bar{z}=\overline{0},-\overline{2}$ so that $\alpha(\pi) \equiv \pi(\bmod M(2))$ or $\alpha(\pi) \equiv-\pi(\bmod M(2))$. The mapping $\psi_{1}: G_{1} \rightarrow h$ defined by $\psi(\alpha)=\rho(\alpha(\pi) / \pi)$ is a homomorphism of $G_{1}$ into $h^{*}$, the multiplicative group of $h$, having kernel $H_{1}$. Thus from above $\psi_{1}\left(G_{1}\right)=\{1,-1\} \subset h^{*}$ so that $G_{1} / H_{1}$ is isomorphic to the group of order 2 .

Proof of Theorem 3. The method of proof is as follows: We 
start with an inertial isomorphism $\alpha_{1}: R \rightarrow R_{2 p}$ and attempt to extend $\alpha_{1}$ to an automorphism on $R_{2 p}$. We define the polynomial $\alpha_{1}(f)(x)$ by

$$
\alpha_{1}(f)(x)=x^{2 p}+p \sum_{i=0}^{2 p-1} \alpha_{1}\left(\alpha_{i}\right) x^{i} .
$$

Then the extension is obtained by constructing a root, $\pi \lambda$, for the polynomial $\alpha_{1}(f)(x)$ and extending $\alpha_{1}$ to an automorphism $\alpha$ on $R_{2 p}$ by defining $\alpha(a)=\alpha_{1}(a)$ for all $a \in R$ and $\alpha(\pi)=\pi \lambda$. In some cases the inertial isomorphism $\alpha_{1}$ must be constructed by using a convergent higher derivation. In others $\alpha_{1}$ may be an arbitrary isomorphism. In either case observe first that if $\alpha_{1}: R \rightarrow R_{2 p}$ is an inertial isomorphism, then if it extends to a nontrivial automorphism $\alpha \in G_{1} \backslash H_{1}$, it must have the property that $\alpha(\pi)=\pi\left(-1+\pi^{n} z\right)$ where $n$ is a positive integer and $z$ is a unit. We assume first that $\rho\left(a_{0}\right) \in h^{p}$ and that we have used Lemma 1.2 to choose a prime element $\pi$ so that $\rho\left(c_{0}\right) \in h^{p}$. Further, let $\alpha(a)=a+\pi \alpha^{*}(a)$ for all $a \in R \backslash M$, and observe that when $t=0$, the $j$ as defined in the theorem is the $s$ defined earlier and when $t>0, s=2 p$. To observe how any extension $\alpha$ of $\alpha_{1}$ must behave we apply it to $f(\pi)$ and simplify to obtain

$$
\begin{aligned}
& \pi^{2 p} \sum_{i}^{2 p}\left(\begin{array}{c}
2 p \\
k
\end{array}\right)(-1)^{2 p-k} \pi^{n k} z^{k}+p \sum_{i=1}^{2 p-1} a_{i} \pi^{i}\left[(-1)^{i}-1\right] \\
& \quad+p \sum_{i=1}^{2 p-1} \pi^{i+1}(-1)^{i} \alpha^{*}\left(a_{i}\right)+p \sum_{i=1}^{2 p-1} a_{i} \pi^{i} \sum_{k=1}^{i}\left(\begin{array}{c}
i \\
k
\end{array}\right)(-1)^{i-k} \pi^{n k} z^{k} \\
& \quad+p^{2} \pi \alpha^{*}\left(c_{1}\right)=0 .
\end{aligned}
$$

Since there is a large number of possible cases that can occur when considering the various possible values that $t, j$, and $n$ may have, and since the methods used in treating them are essentially the same, we will treat only typical cases when $t=0$ and $n=1$. Thus, considering the terms of minimum value in (4.7) we have several cases:

Case 1. $j$ even, $j<p-1$. Then

$$
\alpha^{*}\left(a_{j}\right)-2 a_{j+1}+j z a_{j} \equiv 0 \quad(\bmod M) .
$$

Case 2. $j=p-1$. Then

$$
2 c_{0}^{p} z^{p}+a_{p-1} z-2 a_{p}+\alpha^{*}\left(a_{p-1}\right) \equiv 0 \quad(\bmod M) .
$$

Case 3. $j>p$. Then $\pi^{2 p}\left(\begin{array}{c}2 p \\ p\end{array}\right)(-1)^{p} \pi^{p} z^{p}$ has unique minimum value.

Case 4. $j$ odd, $j<p-1$. Then $p a_{j}(-2) \pi^{j}$ has unique minimum value. 
Case 5. $j=p$. Then

$$
-c_{0}^{p} z^{p}+a_{p} \equiv 0 \quad(\bmod M) .
$$

Part (a) of the theorem follows from consideration of Cases 3, 4, and 5. Considering the remaining cases in more detail, in Case 1 if $\rho\left(a_{j}\right) \in h^{p}$ and $\rho\left(a_{j+1}\right)=0$, then $j z a_{j} \equiv 0(\bmod M)$, which is impossible. However, this case can be further developed by taking $n>1$. If $\rho\left(a_{j}\right) \in h^{p}$ and $\rho\left(a_{j+1}\right) \neq 0$, then in our later construction of $\lambda$ we may choose $z=2 a_{j+1} / j a_{j}$ so that in this case $\alpha_{1}$ may be an arbitrary inertial isomorphism. If $\rho\left(a_{j}\right) \notin h^{p}$, then if $\bar{S}$, is a $p$-basis for $h$, we can assume that $\rho\left(a_{j}\right) \in \bar{S}$ and define a derivation $\delta \in \mathscr{D}(h)$ by choosing $\delta\left(\rho\left(a_{j}\right)\right)$ so that

$$
\delta\left(\rho\left(a_{j}\right)\right)=\rho\left(2 a_{j+1}-j z a_{j}\right)
$$

for whatever choice of $z$ is made later. This $\delta \in \mathscr{D}(h)$ lifts to a $d \in \mathscr{D}(R)$ and we define a higher derivation $E=\left\{E_{i}\right\}$ on $R$ by letting $E_{0}$ be the identity mapping, $E_{1}=d$ and $E_{i}(S)=0$ for $i>1$. Then define $D=\left\{D_{i}\right\}$ by $D_{i}=\pi^{i} E_{i}$. Clearly, $D$ converges on $R$ by Lemma A. Now let $\alpha_{1}=\sum D_{i}$ so that $\alpha_{1}^{*}(\alpha) \equiv D_{1}(a) / \pi(\bmod M)$. In Case 2 if $\rho\left(a_{p-1}\right) \in h^{p}$, then $\rho\left(\alpha^{*}\left(a_{p-1}\right)\right)=0$ so that when $\rho\left(a_{p}\right) \neq 0$, the existence of $\alpha$ is equivalent to the existence of a nontrivial solution $z$ in $h$ for the equation

$$
\rho\left(z^{p}+\frac{a_{p}-1}{2 a_{0}} z-\frac{a_{p}}{a_{0}}\right)=0,
$$

which in this case is equivalent to $R_{2 p} / R$ being Galois. If, however, $\rho\left(a_{p}\right)=0$, then a nontrivial $\alpha \in G_{1} \backslash H_{1}$ can be constructed using $n>1$. If $\rho \notin h^{p}$, we choose a set of representatives $S$ of a $p$-basis $\bar{S}$ for $h$ to contain $\alpha_{p-1}$ and construct an inertial isomorphism $\alpha_{1}: R \rightarrow R_{2 p}$ in the same manner as in Case 1 . Now, starting with an inertial automorphism $\alpha_{1}: R \rightarrow R_{2 p}$, we want to extend $\alpha_{1}$ to an inertial automorphism $\alpha$ on $R_{2 p}$ by constructing a $\lambda \equiv-1(\bmod M)$ such that $\alpha_{1}(f)(\pi \lambda)=0$. We construct such a $\lambda$ by induction in a manner similar to that used in the proof of Theorem 4. Thus, choose $z_{1}$ for $z$ to be a solution, where applicable, to (4.8), (4.9), (4.10) or (4.11). Now letting $\lambda_{1}=\left(-1+\pi z_{1}\right)$, we have that $\alpha_{1}(f)\left(\pi \lambda_{1}\right) \in M(2 p+j+2)$. Now suppose that $z_{k}$ has been chosen so that for $\lambda_{k}=-1+z_{1} \pi+\cdots+z_{k} \pi^{k}$, $\alpha_{1}(f)\left(\pi \lambda_{k}\right) \in M(2 p+k+j+1)$. Then for $\lambda_{k+1}=\lambda_{k}+\pi^{k+1} z_{k+1}$, it is routine to verify that $z_{k+1}$ can be chosen so that $\alpha_{1}(f)\left(\pi \lambda_{k+1}\right) \in$ $M(2 p+k+j+2)$. Let $\lambda=\lim _{k \rightarrow \infty} \lambda_{k}$ so that $\alpha_{1}(f)(\pi \lambda)=0$. Then extend $\alpha_{1}$ to $R_{2 p}$ by defining $\alpha(\pi)=\lambda \pi$.

Now suppose that $\rho\left(a_{0}\right) \notin h^{p}$. As before we assume that $\alpha \in G_{1} \backslash H_{1}$ and determine the properties that $\alpha$ must have. We then construct 
such an $\alpha$ in every case. Thus we assume that $\alpha(a)=a+\pi \alpha^{*}(a)$ for all $a \in R$ and that $\alpha(\pi)=-\pi+\pi^{n} z$ where $z$ is a unit in $R_{2 p}$. Again, note that if $G_{1} \neq H_{1}$ such an $\alpha$ can be chosen for every prime element $\pi$. We apply $\alpha$ to $f(\pi)$ and examine the terms of minimum value in the resulting equation. In every case it is clear that we can construct a higher derivation isomorphism $\alpha_{1}: R \rightarrow R_{2 p}$, using a $p$-basis for $h$ that includes $\rho\left(a_{0}\right)$ as in Case 1 when $\rho\left(a_{0}\right) \in h^{p}$. Similarly, we can extend $\alpha_{1}$ to be an inertial automorphism of $R_{2 p}$ as before. Thus when $\rho\left(a_{0}\right) \notin h^{p}, G_{1} \neq H_{1}$.

Finally, observe that the automorphisms constructed in the above proof cannot be in $G_{D}$ since [5, Lemma 5] requires that $\alpha_{D}(\pi)-\pi \epsilon$ $M(2)$. Moreover, not all of these automorphisms can be Galois since Theorem 5 states that automorphisms of finite order occur only for certain values of $s$. Thus, in general the automorphisms in $G_{1} \backslash H_{1}$ are neither derivation nor Galois automorphisms.

\section{REFERENCES}

1. R. D. Davis and E. Wishart, Galois extensions and the ramification sequence of some wildly ramified $\pi$-adic fields, Proc. Amer. Math Soc., 30 (1971), 212-216.

2. N. Heerema, Derivations on p-adic fields, Trans. Amer. Math. Soc., 102 (1962), 346-351.

3. - Inertial isomorphisms of v-rings, Canad. J. Math., 19 (1967), 529-539.

4. - Inertial automorphisms of a class of wildly ramified v-rings, Trans. Amer. Math. Soc., 132 (1968), 45-54.

5. - Convergent higher derivations on local rings, Trans. Amer. Math. Soc., 132 (1968), 31-44.

6. - Derivations and automorphisms on complete regular local rings, Amer. J. Math., 88 (1966), 33-42.

7. N. Jacobson, Lectures in Abstract Algebra, Vol. III, D. Van Nostrand, Princeton, New Jersey, 1964.

8. S. MacLane, Subfields and automorphism groups of p-adic fields, Ann. Math., 40 (1939), 423-442.

9. J. Neggers, Derivations on $\bar{p}$-adic fields, Trans, Amer. Math. Soc., 115 (1965), 496-504.

10. O. F. G. Schilling, The theory of valuations, Mathematical Surveys No. IV, Amer. Math. Soc., New York, 1950.

11. J. P. Serre, Corps Locaux, Hermann, Paris, 1962.

12. E. Weiss, Algebraic Number Theory, McGraw-Hill, New York, 1963.

13. E. Wishart, Higher derivations on $\bar{p}$-adic fields, $\mathrm{Ph}$. D. Dissertation, Florida State University, 1965.

Received March 18, 1977

UNIVERSITY of NEVADA, RENO, NV 89557 



\section{PACIFIC JOURNAL OF MATHEMATICS}

\section{EDITORS}

RICHARD ARENS (Managing Editor)

University of California

Los Angeles, California 90024

C. W. CurTis

University of Oregon

Eugene, OR 97403

C. C. MOORE

University of California

Berkeley, CA 94720
J. DUGUNDJI

Department of Mathematics University of Southern California Los Angeles, California 90007

R. FinN AND J. Milgram Stanford University Stanford, California 94305

\section{ASSOCIATE EDITORS}

E. F. BECKENBACH

B. H. NeumanN

F. WOLF

K. YoSHIDA

\section{SUPPORTING INSTITUTIONS}

UNIVERSITY OF BRITISH COLUMBIA CALIFORNIA INSTITUTE OF TECHNOLOGY UNIVERSITY OF CALIFORNIA MONTANA STATE UNIVERSITY UNIVERSITY OF NEVADA, RENO NEW MEXICO STATE UNIVERSITY OREGON STATE UNIVERSITY UNIVERSITY OF OREGON
UNIVERSITY OF SOUTHERN CALIFORNIA

STANFORD UNIVERSITY

UNIVERSITY OF HAWAII

UNIVERSITY OF TOKYO

UNIVERSITY OF UTAH

WASHINGTON STATE UNIVERSITY

UNIVERSITY OF WASHINGTON 


\section{Pacific Journal of Mathematics \\ Vol. 78, No. $1 \quad$ March, 1978}

Simeon M. Berman, A class of isotropic distributions in $\mathbf{R}^{n}$ and their

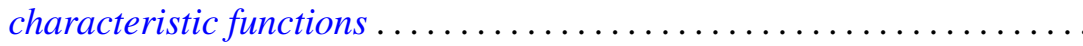

Ezra Brown and Charles John Parry, The 2-class group of biquadratic fields.

II ........................................ 11

Thomas E. Cecil and Patrick J. Ryan, Focal sets of submanifolds ....... 27

Joseph A. Cima and James Warren Roberts, Denting points in $B^{p} \ldots \ldots \ldots 41$

Thomas W. Cusick, Integer multiples of periodic continued fractions . . . . . 47

Robert D. Davis, The factors of the ramification sequence of a class of

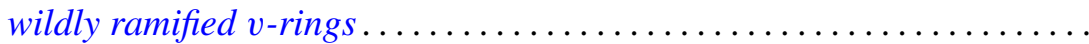

Robert Martin Ephraim, Multiplicative linear functionals of Stein

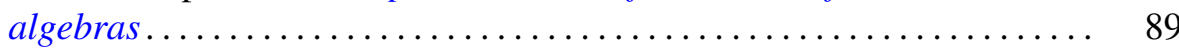

Philip Joel Feinsilver, Operator calculus . .................... 95

David Andrew Gay and William Yslas Vélez, On the degree of the splitting field of an irreducible binomial ..........................

Robert William Gilmer, Jr. and William James Heinzer, On the divisors of

monic polynomials over a commutative ring ..................

Robert E. Hartwig, Schur's theorem and the Drazin inverse .............

Hugh M. Hilden, Embeddings and branched covering spaces for three and four dimensional manifolds ............................

Carlos Moreno, The Petersson inner product and the residue of an Euler product. ...

Christopher Lloyd Morgan, On relations for representations of finite groups....

Ira J. Papick, Finite type extensions and coherence

$\mathrm{R}$. Michael Range, The Carathéodory metric and holomorphic maps on a class of weakly pseudoconvex domains ................

Donald Michael Redmond, Mean value theorems for a class of Dirichlet series

Daniel Reich, Partitioning integers using a finitely generated semigroup ...

Georg Johann Rieger, Remark on a paper of Stux concerning squarefree

numbers in non-linear sequences

Gerhard Rosenberger, Alternierende Produkte in freien Gruppen ..

Ryōtarō Satō, Contraction semigroups in Lebesgue space

Tord Sjödin, Capacities of compact sets in linear subspaces of $\mathbf{R}^{n}$

Robert Jeffrey Zimmer, Uniform subgroups and ergodic actions of exponential Lie groups......................... 